\title{
Optimal and Robust Power Allocation for Visible Light Positioning Systems Under Illumination Constraints
}

\author{
Musa Furkan Keskin ${ }^{\circledR}$, Ahmet Dundar Sezer ${ }^{\circledR}$, and Sinan Gezici ${ }^{\circledR}$, Senior Member, IEEE
}

\begin{abstract}
The problem of optimal power allocation among light emitting diode (LED) transmitters in a visible light positioning system is considered for the purpose of improving localization performance of visible light communication (VLC) receivers. Specifically, the aim is to minimize the Cramér-Rao lower bound (CRLB) on the localization error of a VLC receiver by optimizing LED transmission powers in the presence of practical constraints, such as individual and total power limitations and illuminance constraints. The formulated optimization problem is shown to be convex and thus can efficiently be solved via standard tools. We also investigate the case of imperfect knowledge of localization parameters and develop robust power allocation algorithms by taking into account both overall system uncertainty and individual parameter uncertainties related to the location and orientation of the VLC receiver. In addition, we address the total power minimization problem under predefined accuracy requirements to obtain the most energy-efficient power allocation vector for a given CRLB level. Numerical results illustrate the improvements in localization performance achieved by employing the proposed optimal and robust power allocation strategies over the conventional uniform and non-robust approaches.
\end{abstract}

Index Terms - Visible light positioning, power allocation, robust design, convex optimization, semidefinite programming, iterative entropic regularization.

\section{A. Background and Motivation}

W ITH the advent of low-cost and energy-efficient light emitting diode (LED) technologies, LED based visible light communication (VLC) systems have gathered a significant amount of research interest in the last decade [1]-[3]. Utilizing the vast unlicensed visible light spectrum, VLC has the potential to surmount the issue of spectrum scarcity encountered in radio frequency (RF) based wireless systems [4]. In indoor scenarios, VLC systems can employ the available lighting infrastructure to provide various capabilities simultaneously, such as illumination, high-speed data transmission, and localization [2], [5]. Apart from their basic function of illuminating indoor spaces, LEDs can be

Manuscript received January 22, 2018; revised June 13, 2018 and August 15, 2018; accepted August 18, 2018. Date of publication August 23, 2018; date of current version January 15, 2019. The associate editor coordinating the review of this paper and approving it for publication was A. Khalighi. (Corresponding author: Sinan Gezici.)

The authors are with the Department of Electrical and Electronics Engineering, Bilkent University, 06800 Ankara, Turkey (e-mail: keskin@ ee.bilkent.edu.tr; adsezer@ee.bilkent.edu.tr; gezici@ee.bilkent.edu.tr).

Color versions of one or more of the figures in this paper are available online at http://ieeexplore.ieee.org.

Digital Object Identifier 10.1109/TCOMM.2018.2866849 modulated at high frequencies to accomplish high data rate transmission [3], [6], [7]. On the other hand, the process of localization via visible light signals can be realized by visible light positioning (VLP) systems, where VLC receivers equipped with photo detectors can perform position estimation by exploiting signals emitted by LED transmitters at known locations [5], [7]-[9]. Since line-of-sight (LOS) links generally exist between LED transmitters and VLC receivers, and multipath effects are not very significant as compared to RF based solutions [10], [11], VLP systems can facilitate precise location estimation in indoor environments [9], [12]-[14].

In order to provide satisfactory performance for mobile or stationary devices, it is essential to investigate performance optimization in visible light systems with respect to various criteria, such as mean-squared error (MSE) minimization (e.g., [15]-[18]) and transmission rate maximization (e.g., [19]-[27]). In the literature, transmit precoding and DC offset ${ }^{1}$ designs are extensively explored to improve the MSE performance of multiple-input multiple-output (MIMO) VLC systems [15]-[18]. In addition to transceiver and offset designs in VLC systems, an increasingly popular research strand focuses on power allocation for LED transmitters to enhance system performance [19]-[26], [28], [29]. Due to practical concerns related to energy efficiency and LED lifespan, transmission powers of LEDs in visible light systems are valuable resources that can have profound effects on both transmission rates of VLC systems and localization accuracy of VLP systems. In [19], the total instantaneous data rate of LED arrays is considered as the performance metric for a MIMO VLC system and the optimal strategy for LED power allocation is derived under sum optical power and non-negativity constraints. The studies in [20] and [22] perform power optimization for LEDs to maximize the sum transmission rate of all subcarriers in a VLC system employing optical orthogonal frequency-division multiplexing (OFDM). With the aim of achieving proportional fairness among users in a multi-user VLC network, the total logarithmic throughput is optimized in [23] and [26] to identify the optimal LED power control strategy. Although total and individual power constraints are extensively utilized in power allocation optimization in VLC systems, several studies incorporate color and luminance constraints into the power optimization framework,

\footnotetext{
${ }^{1}$ Optical intensity modulation in VLC systems requires that the amplitude of the electrical drive current of the LED must be non-negative [19].
} 
as well, in compliance with the illumination functionality of VLC systems [21], [24]. In general, power allocation algorithms in both VLC and VLP systems should take into account a variety of design requirements imposed by the multifaceted nature of visible light applications.

The concept of power allocation has also been widely considered for RF based wireless localization networks [30]-[39], where the transmit powers of anchor nodes (the locations of which are known) can be optimized to improve the localization accuracy of target nodes (with unknown locations). The prevailing approach in such investigations is to adopt a mathematically tractable and tight bound on the localization error as the performance metric and to formulate the optimization problem under average and peak anchor power constraints. In [30] and [31], anchor power allocation algorithms are designed to minimize the total power consumption subject to predefined accuracy requirements for localization of target nodes. For cooperative localization networks, distributed power allocation strategies are developed in [32], where the transmit powers of both anchors and targets are optimally allocated to minimize the squared position error bound (SPEB). Moreover, [35] explores the problem of optimal power allocation for OFDM subcarriers in the presence of both perfect and imperfect knowledge of network parameters. As commonly observed in RF wireless localization systems, optimal power allocation provides non-negligible performance benefits over the traditional uniform strategy for a wide range of localization scenarios.

\section{B. Contributions}

Motivated by the promising performance improvements achieved via power allocation in both RF localization networks and VLC systems, we propose the problem of optimal power allocation for LED transmitters in a VLP system, where the objective is to minimize the localization error of the VLC receiver subject to practical constraints related to power and illumination. Leveraging tools from convex optimization and semidefinite programming (SDP), we formulate and solve various optimization problems in both the absence and presence of parameter uncertainties. The power allocation problem for VLP systems has the following key differences from the one in RF based localization systems: (i) Due to the limited linear region of operation, the LEDs are subject to both the minimum and peak power constraints [17], [21], [40], [41]. (ii) Since VLP systems serve the dual purpose of illumination and localization, the problem formulation should include lighting constraints that guarantee an acceptable level of illumination in indoor spaces [41]-[44]. (iii) In contrast to RF systems in which multipath components can severely affect the quality of localization, the received signal power in VLP systems can accurately be characterized by the Lambertian formula [11]

The main contributions of this study can be listed as follows:

- Problem Formulation for LED Power Allocation: For the first time in the literature, we investigate the problem of optimal power allocation among LED transmitters in a VLP system for maximizing the localization accuracy of a VLC receiver. Specifically, we employ the Cramér-Rao lower bound (CRLB) on the localization error as the performance measure and formulate the power allocation problem to minimize the CRLB in the presence of transmission power and illumination constraints.

- Robustness Under Overall System Uncertainty2: We consider the problem of robust power allocation under imperfect knowledge of system parameters and demonstrate that the resulting worst-case CRLB minimization problem can equivalently be transformed into a convex program, which further simplifies to an SDP via constraint relaxation.

- Robustness Under Individual Parameter Uncertainties: We present robust power allocation schemes in the presence of uncertainties in the location and orientation of the VLC receiver. To tackle the resulting intractable optimization problems, we propose an iterative entropic regularization approach where, at each iteration, a convex problem is solved and a three (two)-dimensional grid search is executed over the uncertainty region corresponding to the location (orientation) of the VLC receiver.

- Sum Power Minimization Under Preset Accuracy Constraints: We formulate the minimum power consumption problem under the constraint that the CRLB for localization of the VLC receiver does not exceed a certain threshold. We also extend the problem to the case of overall system uncertainty and prove that the resulting worst-case accuracy constrained optimization problem is shown to be reformulated as a convex one, leading to efficient solutions.

In addition, numerical results show that the proposed optimal power allocation approach for LED transmitters yields significant localization performance gains over the conventional uniform power assignment method. For the case of imperfect knowledge of localization related parameters, the proposed robust power allocation strategies are shown to outperform the uniform and non-robust (which disregards the uncertainty in parameter measurements) strategies.

\section{System MOdeL}

We consider a VLP system with $N_{\mathrm{L}}$ LED transmitters and a VLC receiver, where the objective is to estimate the unknown location of the VLC receiver by utilizing signals emitted by the LED transmitters. As is commonly the case for visible light systems [5], [45], we assume a line-of-sight (LOS) scenario between each LED transmitter and the VLC receiver. Then, the received signal at the VLC receiver due to the $i$ th LED transmitter is formulated as [45]

$$
r_{i}(t)=\alpha_{i} R_{p} s_{i}\left(t-\tau_{i}\right)+\eta_{i}(t)
$$

for $i \in\left\{1, \ldots, N_{\mathrm{L}}\right\}$ and $t \in\left[T_{1, i}, T_{2, i}\right]$, where $T_{1, i}$ and $T_{2, i}$ specify the observation interval for the signal coming from the $i$ th LED transmitter, $\alpha_{i}$ is the optical channel attenuation factor between the $i$ th LED transmitter and the VLC receiver

\footnotetext{
${ }^{2}$ Overall system uncertainty is defined as the uncertainty related to all the system parameters except for the transmit powers and mathematically formulated as a perturbation matrix.
} 
$\left(\alpha_{i}>0\right), R_{p}$ is the responsivity of the photo detector at the VLC receiver, $s_{i}(t)$ is the transmitted signal of the $i$ th LED transmitter, which is nonzero over an interval of $\left[0, T_{s, i}\right]$, $\tau_{i}$ is the TOA of the signal emitted by the $i$ th LED transmitter, and $\eta_{i}(t)$ is zero-mean additive white Gaussian noise with a spectral density level of $\sigma^{2}$. To enable independent processing of signals coming from different LED transmitters, a certain type of multiple access protocol, such as frequencydivision or time-division multiple access [46], [47], can be employed [4]. Thus, the noise processes, $\eta_{1}(t), \ldots, \eta_{N_{\mathrm{L}}}(t)$, are modeled to be independent. In addition, we assume that the VLC receiver has the knowledge of $R_{p}$ and $s_{i}(t)$, $i \in\left\{1, \ldots, N_{\mathrm{L}}\right\}$.

The TOA parameter in (1) can be expressed as

$$
\tau_{i}=\left\|\boldsymbol{l}_{\mathrm{r}}-\boldsymbol{l}_{\mathrm{t}}^{i}\right\| / c+\Delta_{i}
$$

where $c$ is the speed of light, $\Delta_{i}$ denotes the time offset between the clocks of the $i$ th LED transmitter and the VLC receiver, $\boldsymbol{l}_{\mathrm{r}}=\left[\begin{array}{lll}l_{\mathrm{r}, 1} & l_{\mathrm{r}, 2} & l_{\mathrm{r}, 3}\end{array}\right]^{T}$ and $\boldsymbol{l}_{\mathrm{t}}^{i}=\left[\begin{array}{lll}l_{\mathrm{t}, 1}^{i} & l_{\mathrm{t}, 2}^{i} & l_{\mathrm{t}, 3}^{i}\end{array}\right]^{T}$ denote the locations of the VLC receiver and the $i$ th LED transmitter, respectively, and $\left\|\boldsymbol{l}_{\mathrm{r}}-\boldsymbol{l}_{\mathrm{t}}^{i}\right\|$ specifies the distance between the $i$ th LED transmitter and the VLC receiver. For synchronous VLP systems, $\Delta_{i}=0$ for $i=1, \ldots, N_{\mathrm{L}}$ as all the LED transmitters and the VLC receiver are synchronized to a common time reference. On the other hand, for asynchronous systems, where there is no synchronization between the LED transmitters and the VLC receiver, $\Delta_{i}$ 's are modeled as deterministic unknown parameters.

Using the Lambertian model [48], the channel attenuation factor $\alpha_{i}$ in (1) can be written as ${ }^{3}$

$$
\alpha_{i}=-\frac{\left(m_{i}+1\right) S}{2 \pi} \frac{\left[\left(\boldsymbol{l}_{\mathrm{r}}-\boldsymbol{l}_{\mathrm{t}}^{i}\right)^{T} \boldsymbol{n}_{\mathrm{t}}^{i}\right]^{m_{i}}\left(\boldsymbol{l}_{\mathrm{r}}-\boldsymbol{l}_{\mathrm{t}}^{i}\right)^{T} \boldsymbol{n}_{\mathrm{r}}}{\left\|\boldsymbol{l}_{\mathrm{r}}-\boldsymbol{l}_{\mathrm{t}}^{i}\right\|^{m_{i}+3}}
$$

where $m_{i}$ is the Lambertian order for the $i$ th LED transmitter, $S$ is the area of the photo detector at the VLC receiver, and $\boldsymbol{n}_{\mathrm{r}}=\left[\begin{array}{lll}n_{\mathrm{r}, 1} & n_{\mathrm{r}, 2} & n_{\mathrm{r}, 3}\end{array}\right]^{T}$ and $\boldsymbol{n}_{\mathrm{t}}^{i}=\left[\begin{array}{lll}n_{\mathrm{t}, 1}^{i} & n_{\mathrm{t}, 2}^{i} & n_{\mathrm{t}, 3}^{i}\end{array}\right]^{T}$ specify the orientation vectors of the VLC receiver and the $i$ th LED transmitter, respectively [45], [50]. ${ }^{4}$

It is assumed that the parameters $S, \boldsymbol{n}_{\mathrm{r}}, m_{i}, \boldsymbol{l}_{\mathrm{t}}^{i}$, and $\boldsymbol{n}_{\mathrm{t}}^{i}$ for $i=1, \ldots, N_{\mathrm{L}}$ are known by the VLC receiver. For example, the orientation of the VLC receiver, $\boldsymbol{n}_{\mathrm{r}}$, can be measured through a gyroscope and the parameters of the LED transmitters $\left(m_{i}, \boldsymbol{l}_{\mathrm{t}}^{i}\right.$ and $\left.\boldsymbol{n}_{\mathrm{t}}^{i}\right)$ can be transmitted to the receiver via visible light communications. ${ }^{5}$

Remark 1: In VLP systems, the types of signal metrics that can be utilized for position estimation depend on whether the considered system is synchronous or asynchronous. The TOA

\footnotetext{
${ }^{3}$ As the optical channel attenuation $\alpha_{i}$ is non-negative and governs the received signal level, it can be regarded as the received signal strength (RSS) parameter in VLP systems [49].

${ }^{4}$ For example, $\boldsymbol{n}_{\mathrm{r}}=\left[\begin{array}{lll}0 & 0 & 1\end{array}\right]^{T}$ means that the VLC receiver is pointing upwards.

${ }^{5}$ The communication interval between each LED transmitter and the VLC receiver can be divided into two non-overlapping time slots, in which the ranging signals $s_{i}(t)$ can be transmitted in the first one and the parameters of the transmitter can be sent in the second one. The VLC receiver can estimate its own location using the ranging signals and the related parameters transmitted by the LEDs via a consistent LOS link between each LED transmitter and the VLC receiver.
}

parameter in (2) can be used only for synchronous systems as $\Delta_{i}$ 's are unknown in asynchronous scenarios. On the other hand, the RSS parameter in (3) conveys information related to the unknown location of the VLC receiver, $\boldsymbol{l}_{\mathrm{r}}$, in both synchronous and asynchronous VLP systems since it does not depend on the time offsets $\Delta_{i}$ 's. Hence, in synchronous scenarios, both the TOA and RSS parameters can be employed for positioning, whereas in asynchronous scenarios, only the RSS parameter can be used for estimating $l_{\mathrm{r}}$. The analysis in the subsequent sections is generic in the sense that it covers both synchronous VLP systems (which rely on hybrid TOA/RSS based positioning) and asynchronous VLP systems (which utilize only the RSS parameter for positioning).

\section{Optimal Power Allocation For LEDS}

In this section, we establish a theoretical framework for the optimization of LED transmit powers with the aim of maximizing the localization performance of the VLC receiver. First, we describe the optimization variables and the optimization performance metric. Then, by incorporating several practical constraints related to indoor visible light scenarios, we present the formulation of the optimal power allocation problem.

\section{A. Optimization Variables}

The transmitted signal $s_{i}(t)$ from the $i$ th LED transmitter can be expressed as

$$
s_{i}(t)=\sqrt{P_{i}} \widetilde{s}_{i}(t)
$$

for $i \in\left\{1, \ldots, N_{\mathrm{L}}\right\}$, where $\widetilde{s}_{i}(t)$ is a base signal such that $\int_{0}^{T_{s, i}}\left(\widetilde{s}_{i}(t)\right)^{2} d t / T_{s, i}=1$, and $P_{i}$ is a parameter that specifies the transmit power of the $i$ th LED. Then, the optical power of $s_{i}(t)$ can be obtained as [45]

$$
E_{i}^{\mathrm{opt}}=\int_{0}^{T_{s, i}} s_{i}(t) d t / T_{s, i}=\sqrt{P_{i}} \widetilde{E}_{i}^{\mathrm{opt}}
$$

where $T_{s, i}$ denotes the period of $s_{i}(t)$ and $\widetilde{E}_{i}^{\mathrm{opt}}$ is the optical power of $\widetilde{s}_{i}(t)$, defined as

$$
\widetilde{E}_{i}^{\mathrm{opt}} \triangleq \int_{0}^{T_{s, i}} \widetilde{s}_{i}(t) d t / T_{s, i} .
$$

On the other hand, the electrical power consumed by the $i$ th LED, $E_{i}^{\text {elec }}$, is proportional to $P_{i}$ [48]; that is, $E_{i}^{\text {elec }} \propto$ $\int_{0}^{T_{s, i}}\left(s_{i}(t)\right)^{2} d t / T_{s, i}=P_{i}$. In this study, we aim at optimizing the electrical powers of the transmitted signals by adjusting $\left\{P_{i}\right\}_{i=1}^{N_{\mathrm{L}}}$ to maximize the localization performance.

\section{B. Optimization Metric}

The CRLB on the variance of any unbiased estimator $\hat{l}_{\mathrm{r}}$ for the location of the VLC receiver $l_{\mathrm{r}}$ can be expressed as

$$
\mathbb{E}\left\{\left\|\hat{\boldsymbol{l}}_{\mathrm{r}}-\boldsymbol{l}_{\mathrm{r}}\right\|^{2}\right\} \geq \operatorname{trace}\left\{\mathbf{J}^{-1}(\mathbf{p})\right\}
$$

where the Fisher information matrix (FIM) is given by [49]

$$
\mathbf{J}(\mathbf{p})=\left(\mathbf{I}_{3} \otimes \mathbf{p}\right)^{T} \boldsymbol{\Gamma}
$$


with

$$
\begin{aligned}
& \mathbf{p} \triangleq\left[P_{1} \ldots P_{N_{\mathrm{L}}}\right]^{T} \in \mathbb{R}^{N_{\mathrm{L}}} \\
& \boldsymbol{\Gamma} \triangleq\left[\begin{array}{lll}
\gamma_{1,1} & \gamma_{1,2} & \gamma_{1,3} \\
\gamma_{2,1} & \gamma_{2,2} & \gamma_{2,3} \\
\gamma_{3,1} & \gamma_{3,2} & \gamma_{3,3}
\end{array}\right] \in \mathbb{R}^{3 N_{\mathrm{L}} \times 3} \\
& \gamma_{k_{1}, k_{2}} \triangleq\left[\gamma_{k_{1}, k_{2}}^{(1)} \ldots \gamma_{k_{1}, k_{2}}^{\left(N_{\mathrm{L}}\right)}\right]^{T} \in \mathbb{R}^{N_{\mathrm{L}}}
\end{aligned}
$$

for $k_{1}, k_{2} \in\{1,2,3\} . \mathbf{I}_{3}$ and $\otimes$ in (8) represents, respectively, the $3 \times 3$ identity matrix and the Kronecker product, $P_{i}$ in (9) is as defined in Section III-A and $\gamma_{k_{1}, k_{2}}^{(i)}$ in (11) is given by Appendix A. ${ }^{6}$

We employ the CRLB in (7) as the optimization performance metric for quantifying the localization accuracy of the VLC receiver. ${ }^{7}$ The reason for this choice is that the maximum likelihood (ML) estimator for the location of the VLC receiver can attain the CRLB for sufficiently high signal-to-noise ratios (SNRs) [51], [52]. In addition, the CRLB expression facilitates theoretical analyses and results in mathematically tractable formulations. Also, the usage of the CRLB as a performance measure renders the analysis independent of any specific location estimator, thereby providing a generic framework for power allocation in VLP systems.

\section{VLP System Constraints}

Certain constraints must be imposed on a VLP system while designing LED power optimization schemes in order to satisfy illumination, energy, and hardware related requirements. In particular, the following system constraints are taken into account in the power optimization problem:

1) Individual Power Constraints: Lower and upper bound constraints for LED powers must be incorporated to ensure that transmission powers of LEDs lie inside the linear region of operation so that the LED output power is proportional to the input drive current, which provides efficient electrical-tooptical conversion [17], [21], [40], [41], [53]. In addition, selfheating induced by a high drive current may shorten the LED lifetime [54]. Hence, the resulting constraint set is given by

$$
\mathcal{P}_{1} \triangleq\left\{\mathbf{p} \in \mathbb{R}^{N_{\mathrm{L}}}: \mathbf{p}_{\mathrm{lb}} \preceq \mathbf{p} \preceq \mathbf{p}_{\mathrm{ub}}\right\}
$$

where $\mathbf{p}_{\mathrm{lb}} \in \mathbb{R}^{N_{\mathrm{L}}}$ and $\mathbf{p}_{\mathrm{ub}} \in \mathbb{R}^{N_{\mathrm{L}}}$ denote, respectively, the lower and upper bounds on $\mathbf{p}$ in (9).

2) Total Power Constraint: Due to power consumption restrictions of LEDs and human eye safety considerations, the total electrical power of LEDs in a VLP system must be limited [22], [41], [48], [55]. Therefore, we have the following constraint set regarding the total power limit:

$$
\mathcal{P}_{2} \triangleq\left\{\mathbf{p} \in \mathbb{R}^{N_{\mathrm{L}}}: \mathbf{1}^{T} \mathbf{p} \leq P_{\mathrm{T}}\right\}
$$

${ }^{6}$ The FIM expression $\mathbf{J}(\mathbf{p})$ in (8) comprises both synchronous and asynchronous VLP scenarios, i.e., it represents both hybrid TOA/RSS based positioning and RSS-only positioning, with the difference being reflected in $\gamma_{k_{1}, k_{2}}^{(i)}$ in (11). Please see (64)-(66) in Appendix A to see how $\gamma_{k_{1}, k_{2}}^{(i)}$ differs between synchronous and asynchronous cases.

${ }^{7}$ It should be emphasized that this performance metric constitutes a lower bound on the positioning error of unbiased estimators and therefore may not be the same as the actual positioning error. However, as will be shown in Section VII-B, the optimization based on the CRLB leads to a similar level of improvement on the RMSE performance of the ML estimators. where $P_{\mathrm{T}}$ determines the total electrical power constraint of LEDs.

3) Individual Illumination Constraints: Since VLP systems are utilized also for indoor lighting in addition to other benefits such as data transmission and localization, a certain level of brightness must be maintained over the room and/or at specified locations [41]-[44]. The illuminance $\left(\mathrm{lm} / \mathrm{m}^{2}, \mathrm{~lx}\right)$ is used as a measure of brightness, which is defined as the luminous flux (lm) per unit area [56]. Combining [44, eq. (3)], [56, eq. (16.3)] and (5), the horizontal illuminance at location $\mathbf{x}$ generated by the $i$ th LED can be calculated as

$$
\mathcal{I}_{\text {ind }}^{i}\left(\mathbf{x}, P_{i}\right)=\sqrt{P_{i}} \phi_{i}(\mathbf{x})
$$

where

$$
\phi_{i}(\mathbf{x}) \triangleq \frac{\left(m_{i}+1\right) \kappa_{i} \widetilde{E}_{i}^{\mathrm{opt}}}{2 \pi} \frac{\left[\left(\mathbf{x}-\boldsymbol{l}_{\mathrm{t}}^{i}\right)^{T} \boldsymbol{n}_{\mathrm{t}}^{i}\right]^{m_{i}}\left(l_{\mathrm{t}, 3}^{i}-x_{3}\right)}{\left\|\mathbf{x}-\boldsymbol{l}_{\mathrm{t}}^{i}\right\|^{m_{i}+3}}
$$

with $\widetilde{E}_{i}^{\text {opt }}$ being as defined in (6) and $\kappa_{i}$ denoting the luminous efficacy $(\mathrm{lm} / \mathrm{W})$ of the $i$ th LED, defined as the optical power to luminous flux conversion efficiency [56]. Then, the total illuminance at $\mathrm{x}$ produced by all the LEDs can be obtained as follows [57]:

$$
\mathcal{I}_{\text {ind }}(\mathbf{x}, \mathbf{p})=\sum_{i=1}^{N_{\mathrm{L}}} \mathcal{I}_{\text {ind }}^{i}\left(\mathbf{x}, P_{i}\right)=\sum_{i=1}^{N_{\mathrm{L}}} \sqrt{P_{i}} \phi_{i}(\mathbf{x})
$$

Let $L$ denote the number of locations at which the illuminance constraint is to be satisfied. Then, the corresponding constraint set can be defined as

$$
\mathcal{P}_{3} \triangleq\left\{\mathbf{p} \in \mathbb{R}^{N_{\mathrm{L}}}: \mathcal{I}_{\text {ind }}\left(\mathbf{x}_{\ell}, \mathbf{p}\right) \geq \tilde{\mathcal{I}}_{\ell}, \ell=1, \ldots, L\right\}
$$

where $\widetilde{\mathcal{I}}_{\ell}$ is the illuminance constraint defined for location $\mathbf{x}_{\ell}$.

4) Average Illumination Constraint: The expression in (16) quantifies the illuminance level at a specified location in the room. It may also be necessary to keep the average illuminance over the room above a certain threshold to comply with average brightness requirements. Then, the average illuminance is

$$
\mathcal{I}_{\text {avg }}(\mathbf{p})=\sum_{i=1}^{N_{\mathrm{L}}} \sqrt{P_{i}} \frac{\int_{\mathcal{A}} \phi_{i}(\mathbf{x}) d \mathbf{x}}{|\mathcal{A}|}
$$

where $\mathcal{A}$ denotes the region where the average illuminance constraint must be satisfied and $|\mathcal{A}|$ denotes the volume of $\mathcal{A}$. The constraint set associated with the average illuminance is given by

$$
\mathcal{P}_{4} \triangleq\left\{\mathbf{p} \in \mathbb{R}^{N_{\mathrm{L}}}: \mathcal{I}_{\text {avg }}(\mathbf{p}) \geq \widetilde{\mathcal{I}}_{\text {avg }}\right\}
$$

where $\widetilde{\mathcal{I}}_{\text {avg }}$ is the average illuminance constraint.

Remark 2: In addition to the lower bounds on the illumination levels in (17) and (19), we can also impose upper bound constraints to alleviate eye safety risks in human environments. However, since the total power constraint in (13) effectively limits the illumination level, an additional upper bound constraint in (17) and (19) is not required. To express this observation in a formal manner, let $\left\{\widetilde{\mathcal{I}}_{\ell}^{\text {ub }}\right\}_{\ell=1}^{L}$ and $\widetilde{\mathcal{I}}_{\text {avg }}^{\text {ub }}$ denote the upper bounds on the individual and average illuminance levels in (17) and (19), respectively. Then, it follows from the 
Cauchy-Schwarz inequality that these upper bound constraints are satisfied if we choose the total power limit in (13) as

$$
P_{\mathrm{T}} \leq \min \left\{\min _{\ell \in\{1, \ldots, L\}} \frac{\left(\widetilde{\mathcal{I}}_{\ell}^{\mathrm{ub}}\right)^{2}}{\left\|\boldsymbol{\phi}\left(\mathbf{x}_{\ell}\right)\right\|^{2}}, \frac{\left(\widetilde{\mathcal{I}}_{\mathrm{avg}}^{\mathrm{ub}}\right)^{2}}{\|\boldsymbol{\varphi}\|^{2}}\right\},
$$

where $\phi(\mathbf{x})=\left[\phi_{1}(\mathbf{x}) \ldots \phi_{N_{\mathrm{L}}}(\mathbf{x})\right]^{T}$ and $\varphi=\left[\varphi_{1} \ldots \varphi_{N_{\mathrm{L}}}\right]^{T}$ with $\varphi_{i} \triangleq \frac{\int_{\mathcal{A}} \phi_{i}(\mathbf{x}) d \mathbf{x}}{|\mathcal{A}|}$ for $i \in\left\{1, \ldots, N_{\mathrm{L}}\right\}$.

\section{Problem Formulation}

Considering the optimization metric in Section III-B and the system constraints in Section III-C, the problem of optimal power allocation for LED transmitters can be formulated as follows:

$$
\begin{aligned}
& \underset{\mathbf{p}}{\operatorname{minimize}} \operatorname{trace}\left\{\mathbf{J}^{-1}(\mathbf{p})\right\} \\
& \text { subject to } \mathbf{p} \in \mathcal{P}
\end{aligned}
$$

where $\mathcal{P} \triangleq \bigcap_{i=1}^{4} \mathcal{P}_{i}$ and $\mathbf{J}(\mathbf{p})$ is given by (8). In the proposed power optimization framework in (21), we search for the optimal power vector that minimizes the CRLB for the localization of the VLC receiver subject to power and illumination constraints. ${ }^{8}$ The following lemma establishes the convexity of (21).

Lemma 1: The optimization problem in (21) is convex.

Proof: First, the convexity of $f(\mathbf{p}) \triangleq \operatorname{trace}\left\{\mathbf{J}^{-1}(\mathbf{p})\right\}$ in $\mathbf{p}$ is shown as follows: Consider any $\mathbf{p}_{1} \in \mathbb{R}^{N_{\mathrm{L}}}, \mathbf{p}_{2} \in \mathbb{R}^{N_{\mathrm{L}}}$, and $\lambda \in[0,1]$. Then,

$$
\begin{aligned}
& f\left(\lambda \mathbf{p}_{1}+(1-\lambda) \mathbf{p}_{2}\right) \\
& =\operatorname{trace}\left\{\left(\left[\mathbf{I}_{3} \otimes\left(\lambda \mathbf{p}_{1}+(1-\lambda) \mathbf{p}_{2}\right)\right]^{T} \boldsymbol{\Gamma}\right)^{-1}\right\} \\
& =\operatorname{trace}\left\{\left(\lambda\left(\mathbf{I}_{3} \otimes \mathbf{p}_{1}\right)^{T} \boldsymbol{\Gamma}+(1-\lambda)\left(\mathbf{I}_{3} \otimes \mathbf{p}_{2}\right)^{T} \boldsymbol{\Gamma}\right)^{-1}\right\} \\
& \leq \lambda f\left(\mathbf{p}_{1}\right)+(1-\lambda) f\left(\mathbf{p}_{2}\right)
\end{aligned}
$$

where (22) follows from (8), (24) is the result of the properties of Kronecker product, and (25) is due to the convexity of trace $\left\{\mathbf{X}^{-1}\right\}$ for $\mathbf{X} \succ 0$ [58]. Since the constraint sets $\mathcal{P}_{1}$ in (12) and $\mathcal{P}_{2}$ in (13) are linear, and $\mathcal{P}_{3}$ in (17) and $\mathcal{P}_{4}$ in (19) are convex due to the concavity of (16) and (18) with respect to $\mathbf{p}$, the combined constraint set $\mathcal{P}$ is convex, thus proving the convexity of (21) in $\mathbf{p}$.

Based on Lemma 1, it is noted that optimal LED power allocation strategies can be obtained via standard convex optimization tools [58], [59].

\section{Robust Power Allocation With OVERALL SYSTEM UNCERTAINTY}

In Section III, the optimal power allocation is performed by assuming perfect knowledge of localization parameters, which however may not be realistic for practical VLP scenarios.

\footnotetext{
${ }^{8}$ With the optimal power allocation strategy in (21), the available resources of the LED transmitters can be employed to provide improved localization accuracy and a desired level of illumination simultaneously (i.e., no extra power resources are needed for localization in addition to those utilized for illumination).
}

In this section, robust optimization schemes will be designed for power allocation among LED transmitters in the presence of overall uncertainty in VLP system parameters. ${ }^{9}$ In the following, we present the problem formulation for robust power allocation with overall system uncertainty in VLP scenarios and demonstrate that it can be reformulated as a convex optimization problem, which can further be simplified to an SDP via feasible set relaxations.

\section{A. Problem Statement}

Considering the optimization problem in (21), the matrix $\Gamma$ in (10) contains all the information required for LED power optimization based on (8). Since the knowledge of localization related parameters is imperfect in practice, it is assumed that $\boldsymbol{\Gamma}$ is measured with some uncertainty; that is,

$$
\widehat{\Gamma}=\Gamma+\Delta \Gamma
$$

where $\widehat{\Gamma}$ is the estimated/nominal matrix and $\Delta \boldsymbol{\Gamma}$ represents the error matrix that accumulates all the uncertainties in localization parameters. As in [60]-[63], a deterministically bounded error model is considered for $\Delta \Gamma$, i.e.,

$$
\Delta \boldsymbol{\Gamma} \in \mathcal{E} \triangleq\left\{\Delta \boldsymbol{\Gamma} \in \mathbb{R}^{3 N_{\mathrm{L}} \times 3}:\|\Delta \boldsymbol{\Gamma}\| \leq \delta\right\}
$$

for a known size of uncertainty region $\delta$, where $\|\cdot\|$ stands for the matrix spectral norm.

For the robust counterpart of (21), the aim is to minimize the worst-case CRLB over all uncertainties in the form of $\|\Delta \Gamma\| \leq \delta$. Hence, considering the error model in (26), the robust min-max design problem corresponding to the CRLB optimization in (21) can be stated as follows:

$$
\begin{aligned}
& \underset{\mathbf{p}}{\operatorname{minimize}} \max _{\boldsymbol{\Delta} \boldsymbol{\Gamma} \in \mathcal{E}} \operatorname{trace}\left\{\left(\left(\mathbf{I}_{3} \otimes \mathbf{p}\right)^{T}(\widehat{\boldsymbol{\Gamma}}-\boldsymbol{\Delta} \boldsymbol{\Gamma})\right)^{-1}\right\} \\
& \text { subject to } \mathbf{p} \in \mathcal{P}
\end{aligned}
$$

where $\mathcal{E}$ is as defined in (27) and $\mathcal{P}$ is the feasible region in $(21 b)$.

Remark 3: As observed from (10), (11) and (64)-(71), the uncertainty in $\boldsymbol{\Gamma}$ (equivalently, the uncertainty in $\gamma_{k_{1}, k_{2}}^{(i)}$ in (64)) may result from an imperfect knowledge of the following VLP system parameters: $R_{p}, \boldsymbol{l}_{\mathrm{r}}, \boldsymbol{n}_{\mathrm{r}}, \boldsymbol{l}_{\mathrm{t}}^{i}, \boldsymbol{n}_{\mathrm{t}}^{i}$ and $m_{i}$. The maximum possible errors in estimating these parameters can be obtained by utilizing the characteristics of the devices on the LED transmitters and the VLC receiver. For instance, orientation measurement error bounds can be figured out via auxiliary sensor (e.g., gyroscope, accelerometer, magnetometer) properties, while the responsivity of the photodetector and the Lambertian order of the LED transmitters can be determined in a bounded interval from device characteristics. In addition, the uncertainty in $\boldsymbol{l}_{\mathrm{r}}$ can be estimated from the tracking error covariance matrix. Hence, it is reasonable to assume that the uncertainty in $\gamma_{k_{1}, k_{2}}^{(i)}$ is bounded and known. Denoting this uncertainty level by $\varepsilon_{k_{1}, k_{2}}^{(i)}$ (i.e., $\left|\widehat{\gamma}_{k_{1}, k_{2}}^{(i)}-\gamma_{k_{1}, k_{2}}^{(i)}\right| \leq$ $\varepsilon_{k_{1}, k_{2}}^{(i)}$ where $\widehat{\gamma}_{k_{1}, k_{2}}^{(i)}$ represents the estimated value of $\gamma_{k_{1}, k_{2}}^{(i)}$ ),

\footnotetext{
${ }^{9}$ The meaning of overall uncertainty will be clarified in Section IV-A.
} 
we have

$$
\begin{aligned}
\|\boldsymbol{\Delta} \boldsymbol{\Gamma}\| & \leq\|\boldsymbol{\Delta} \boldsymbol{\Gamma}\|_{F}=\|\widehat{\boldsymbol{\Gamma}}-\boldsymbol{\Gamma}\|_{F} \\
& =\left(\sum_{k_{1}=1}^{3} \sum_{k_{2}=1}^{3} \sum_{i=1}^{N_{\mathrm{L}}}\left(\widehat{\gamma}_{k_{1}, k_{2}}^{(i)}-\gamma_{k_{1}, k_{2}}^{(i)}\right)^{2}\right)^{1 / 2} \\
& \leq\left(\sum_{k_{1}=1}^{3} \sum_{k_{2}=1}^{3} \sum_{i=1}^{N_{\mathrm{L}}}\left(\varepsilon_{k_{1}, k_{2}}^{(i)}\right)^{2}\right)^{1 / 2} \triangleq \delta
\end{aligned}
$$

where $\|\cdot\|_{F}$ is the matrix Frobenius norm. Therefore, in practice, the uncertainty level $\delta$ in (27) can be computed via (29)-(31).

Remark 4: The motivation for studying the overall system uncertainty comes from its mathematical tractability that allows for the characterization of uncertainties in a large number of individual parameters (e.g, locations, orientations and other device properties) as a single perturbation/error matrix whose spectral norm is upper bounded by a known value. This uncertainty model has also been used in the localization literature (e.g., [63]) and can lead to computationally favorable robust problem formulations, as will be shown in Section IV-B. The difference between the overall system uncertainty and individual parameter uncertainties (which will be investigated in Section V) is that the former one reflects the combined effect of all the uncertainties in individual parameters using a conservative error model, i.e., via a matrix perturbation model (as in [63, Sec. III-C]) that involves the aggregate uncertainty, while the latter one exploits the specific characteristics of the individual uncertainty regions (e.g., spherical uncertainty set for the location in (44) or bounded angular deviations in orientation measurements in (48)) for achieving robustness in localization (i.e., each individual parameter is considered separately). In cases where it is difficult to handle the uncertainty sets collectively for all individual parameters (note that $\gamma_{k_{1}, k_{2}}^{(i)}$, defined in (64)-(71), is a very complicated expression in terms of the uncertainty sources, e.g., $R_{p}, \boldsymbol{l}_{\mathrm{r}}, \boldsymbol{n}_{\mathrm{r}}, \boldsymbol{l}_{\mathrm{t}}^{i}, \boldsymbol{n}_{\mathrm{t}}^{i}$ and $m_{i}$ [64]), the overall uncertainty model can be especially beneficial in facilitating simultaneous treatment of individual uncertainties. Therefore, it should be emphasized that the individual parameter uncertainties in Section $\mathrm{V}$ are not the special cases of the overall system uncertainty in Section IV; rather, they allow us to investigate the effect of each uncertain parameter on the localization performance independently by itself [64].

\section{B. Equivalent Convex Reformulation of (28)}

The problem in (28) is challenging to solve in its current form and its direct solution is computationally prohibitive. In the following proposition, we demonstrate that (28) can be reformulated as a convex program.

Proposition 1: The robust power allocation problem in (28) can equivalently be expressed as the following convex optimization problem:

$$
\begin{aligned}
& \underset{\mathbf{p}, t, \mathbf{H}, s, \mu}{\operatorname{minimize}} t \\
& \text { subject to } \operatorname{trace}\{\mathbf{H}\} \leq t-d s \\
& \mathbf{\Phi}(\mathbf{p}, \mathbf{H}, s, \mu) \succeq 0
\end{aligned}
$$

$$
\begin{aligned}
& \mathbf{H} \succeq 0, \quad \mu \geq 0 \\
& \mathbf{p} \in \mathcal{P}
\end{aligned}
$$

where

$$
\begin{aligned}
& \boldsymbol{\Phi}(\mathbf{p}, \mathbf{H}, s, \mu) \\
& \triangleq\left[\begin{array}{ccc}
\mathbf{H}+s \mathbf{I} & \mathbf{I} & \mathbf{0} \\
\mathbf{I} & \left(\mathbf{I}_{3} \otimes \mathbf{p}\right)^{T} \widehat{\boldsymbol{\Gamma}}-\mu \mathbf{I} & -\frac{\delta}{2}\left(\mathbf{I}_{3} \otimes \mathbf{p}\right)^{T} \\
\mathbf{0} & -\frac{\delta}{2}\left(\mathbf{I}_{3} \otimes \mathbf{p}\right) & \mu \mathbf{I}
\end{array}\right]
\end{aligned}
$$

and $d$ is the dimension of localization.

Proof: We utilize the following lemmas for the proof [61].

Lemma 2 (18c in [65]): Let $\mathbf{X} \in \mathbb{R}^{d \times d}$ be a symmetric matrix. Then, trace $\{\mathbf{X}\} \leq t$ if and only if there exists $s \in \mathbb{R}$ and $\mathbf{H} \in \mathbb{R}^{d \times d}$ such that

$$
\operatorname{trace}\{\mathbf{H}\} \leq t-d s, \mathbf{H} \succeq 0, \mathbf{H}+s \mathbf{I} \succeq \mathbf{X} .
$$

Lemma 3 (Lemma 2 in [60]): For matrices $\mathbf{A}, \mathbf{B}$ and $\mathbf{C}$ with $\mathbf{A}=\mathbf{A}^{T}$, the matrix inequality

$$
\mathbf{A} \succeq \mathbf{B}^{T} \mathbf{X} \mathbf{C}+\mathbf{C}^{T} \mathbf{X}^{T} \mathbf{B}, \quad \forall \mathbf{X}:\|\mathbf{X}\| \leq \delta
$$

is satisfied if and only if there exists $a \mu \geq 0$ such that

$$
\left[\begin{array}{cc}
\mathbf{A}-\mu \mathbf{C}^{T} \mathbf{C} & -\delta \mathbf{B}^{T} \\
-\delta \mathbf{B} & \mu \mathbf{I}
\end{array}\right] \succeq 0 .
$$

By introducing a slack variable $t,(28)$ can equivalently be written in the epigraph form as follows:

$$
\begin{aligned}
& \underset{\mathbf{p}, t}{\operatorname{minimize}} t \\
& \text { subject to } \operatorname{trace}\left\{\left(\left(\mathbf{I}_{3} \otimes \mathbf{p}\right)^{T}(\widehat{\boldsymbol{\Gamma}}-\boldsymbol{\Delta} \boldsymbol{\Gamma})\right)^{-1}\right\} \leq t, \\
& \qquad \mathbf{p} \in \mathcal{P} \\
& \forall \boldsymbol{\Delta} \boldsymbol{\Gamma}: \boldsymbol{\Delta} \boldsymbol{\Gamma} \in \mathcal{E}
\end{aligned}
$$

First, using Lemma 2 for the constraint in (37b) leads to the following inequalities:

$$
\begin{aligned}
\operatorname{trace}\{\mathbf{H}\} & \leq t-d s, \mathbf{H} \succeq 0 \\
\mathbf{H}+s \mathbf{I} & \succeq\left(\left(\mathbf{I}_{3} \otimes \mathbf{p}\right)^{T}(\widehat{\boldsymbol{\Gamma}}-\boldsymbol{\Delta} \boldsymbol{\Gamma})\right)^{-1}, \quad \forall \boldsymbol{\Delta} \boldsymbol{\Gamma}: \Delta \boldsymbol{\Gamma} \in \mathcal{E}
\end{aligned}
$$

for some $s \in \mathbb{R}$ and $\mathbf{H} \in \mathbb{R}^{d \times d}$. Next, applying the Schur complement lemma to (38b), we have

$$
\left[\begin{array}{cc}
\mathbf{H}+s \mathbf{I} & \mathbf{I} \\
\mathbf{I} & \left(\mathbf{I}_{3} \otimes \mathbf{p}\right)^{T}(\widehat{\boldsymbol{\Gamma}}-\boldsymbol{\Delta} \boldsymbol{\Gamma})
\end{array}\right] \succeq 0, \quad \forall \boldsymbol{\Delta} \boldsymbol{\Gamma}: \boldsymbol{\Delta} \boldsymbol{\Gamma} \in \mathcal{E} .
$$

Rearranging (39), an inequality of the form (35) is obtained as

$$
\begin{array}{cc}
{\left[\begin{array}{cc}
\mathbf{H}+s \mathbf{I} & \mathbf{I} \\
\mathbf{I} & \left(\mathbf{I}_{3} \otimes \mathbf{p}\right)^{T} \widehat{\boldsymbol{\Gamma}}
\end{array}\right] \succeq \mathbf{B}^{T} \boldsymbol{\Delta} \mathbf{\Gamma} \mathbf{C}+\mathbf{C}^{T} \boldsymbol{\Delta} \boldsymbol{\Gamma}^{T} \mathbf{B},} \\
\forall \boldsymbol{\Delta} \boldsymbol{\Gamma}: \boldsymbol{\Delta} \boldsymbol{\Gamma} \in \mathcal{E}
\end{array}
$$

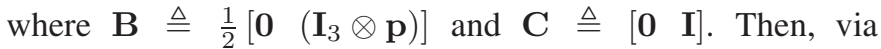
Lemma 3, (40) is transformed into the constraint in (32c), which completes the proof. 


\section{SDP Formulation via Feasible Set Relaxation}

Since (32c) is a linear matrix inequality (LMI) in the variables $\mathbf{p}, \mathbf{H}, s$ and $\mu$ [66], the problem in (32) is convex. In addition, if the general convex constraint (32e) can be relaxed to a linear one by replacing $\mathcal{P}$ with an appropriate $\widetilde{\mathcal{P}}$ satisfying $\widetilde{\mathcal{P}} \supseteq \mathcal{P}$, (32) simplifies to an SDP with a linear objective and a set of LMI constraints [67]. By squaring both sides of (17) and applying the arithmetic mean-geometric mean inequality, a relaxed version of $\mathcal{P}_{3}$ is obtained as

$$
\widetilde{\mathcal{P}}_{3} \triangleq\left\{\mathbf{p}: \boldsymbol{\phi}(\mathbf{x})^{T} \mathbf{p} \geq \widetilde{\mathcal{I}}_{\ell}^{2} / \mathbf{1}^{T} \boldsymbol{\phi}(\mathbf{x}), \quad \ell=1, \ldots, L\right\} \supseteq \mathcal{P}_{3}
$$

where $\phi(\mathbf{x})=\left[\phi_{1}(\mathbf{x}) \ldots \phi_{N_{\mathrm{L}}}(\mathbf{x})\right]^{T}$. Similarly, $\mathcal{P}_{4}$ in (19) can be relaxed to

$$
\widetilde{\mathcal{P}}_{4} \triangleq\left\{\mathbf{p}: \varphi^{T} \mathbf{p} \geq \widetilde{\mathcal{I}}_{\text {avg }}^{2} / \mathbf{1}^{T} \varphi\right\} \supseteq \mathcal{P}_{4}
$$

where $\varphi=\left[\varphi_{1} \ldots \varphi_{N_{\mathrm{L}}}\right]^{T}$ with $\varphi_{i} \triangleq \frac{\int_{\mathcal{A}} \phi_{i}(\mathbf{x}) d \mathbf{x}}{|\mathcal{A}|}$. Hence, by defining $\widetilde{\mathcal{P}} \triangleq \mathcal{P}_{1} \cap \mathcal{P}_{2} \cap \widetilde{\mathcal{P}}_{3} \cap \widetilde{\mathcal{P}}_{4}$ and replacing $\mathcal{P}$ with $\widetilde{\mathcal{P}}$ in (32e), (32) becomes an SDP problem and thus can be solved very efficiently using available convex optimization softwares [59], [68]. The worst-case complexity of an SDP with $n$ variables and $m$ constraints is given by $\mathcal{O}\left(\max (m, n)^{4} n^{1 / 2} \log (1 / \epsilon)\right)$, where $\epsilon$ is the tolerance level [69]. Thus, the computational complexity of the SDP version of (32), which is obtained from the feasible set relaxations, can be expressed as $\mathcal{O}\left(N_{\mathrm{L}}^{4.5} \log (1 / \epsilon)\right)$.

\section{Robust Power Allocation With INDIVIDUal PARAMETER UNCERTAINTIES}

In this section, we consider robust power allocation schemes under individual uncertainties related to localization parameters in VLP systems. In indoor tracking applications, VLC receiver position $l_{\mathrm{r}}$ can be predicted to lie in a validation region, but its exact position cannot perfectly be known. Similarly, VLC receiver orientation $\boldsymbol{n}_{\mathrm{r}}$ may be subject to measurement errors since the measurement devices such as gyroscopes tend to generate noisy parameter estimates. Hence, individual parameter uncertainties must be taken into account while deriving optimal strategies for LED power allocation. In the following, we first present the problem formulations in the presence of uncertainties in the location and the orientation of the VLC receiver. Then, we propose an iterative approach to solve the resulting intractable optimization problems.

\section{A. Uncertainty in VLC Receiver Location}

To formulate the robust power allocation problem in the presence of uncertainties about the location of the VLC receiver, we assume that the nominal location $\hat{l}_{\mathrm{r}}$ is a perturbed version of the true location $l_{\text {r }}$, i.e.,

$$
\hat{l}_{\mathrm{r}}=l_{\mathrm{r}}+\mathbf{e}_{l_{\mathrm{r}}} .
$$

As in [61], [64], and [70], we assume a spherical uncertainty set for the location errors, i.e.,

$$
\mathbf{e}_{l_{\mathrm{r}}} \in \mathcal{E}_{\boldsymbol{l}_{\mathrm{r}}} \triangleq\left\{\mathbf{e} \in \mathbb{R}^{3}:\|\mathbf{e}\| \leq \delta_{\boldsymbol{l}_{\mathrm{r}}}\right\}
$$

where $\delta_{l_{\mathrm{r}}}$ is a known value that represents the size of the uncertainty region. Then, the power allocation problem in (21) based on worst-case CRLB minimization can be formulated as

$$
\begin{aligned}
& \underset{\mathbf{p}}{\operatorname{minimize}} \max _{\mathbf{e}_{l_{\mathrm{r}} \in \mathcal{E}_{\boldsymbol{l}_{\mathrm{r}}}}} \operatorname{trace}\left\{\left(\left(\mathbf{I}_{3} \otimes \mathbf{p}\right)^{T} \boldsymbol{\Gamma}\left(\hat{\boldsymbol{l}}_{\mathrm{r}}-\mathbf{e}_{\boldsymbol{l}_{\mathrm{r}}}\right)\right)^{-1}\right\} \\
& \text { subject to } \mathbf{p} \in \mathcal{P}
\end{aligned}
$$

where $\boldsymbol{\Gamma}\left(\hat{\boldsymbol{l}}_{\mathrm{r}}-\mathbf{e}_{l_{\mathrm{r}}}\right)$ denotes the matrix $\boldsymbol{\Gamma}$ in (10) evaluated at $l_{\mathrm{r}}=\hat{l}_{\mathrm{r}}-\mathbf{e}_{l_{\mathrm{r}}}$.

\section{B. Uncertainty in VLC Receiver Orientation}

The orientation vector of the VLC receiver can be expressed as

$$
\boldsymbol{n}_{\mathrm{r}}(\theta, \phi)=[\sin \theta \cos \phi \sin \theta \sin \phi \cos \theta]^{T}
$$

where $\theta$ and $\phi$ represent the polar and the azimuth angles, respectively [64]. According to (46), the uncertainty related to the orientation of the VLC receiver can be modeled as angular uncertainties in $\theta$ and $\phi$ [64]. Hence, the nominal (measured) polar and azimuth angles can be written as

$$
\hat{\theta}=\theta+e_{\theta}, \quad \hat{\phi}=\phi+e_{\phi}
$$

where $\theta$ and $\phi$ are the true values of the angles, and $e_{\theta}$ and $e_{\phi}$ represent the errors in angular measurements, for which the bounded uncertainty sets can be defined as

$$
\begin{aligned}
& e_{\theta} \in \mathcal{E}_{\theta} \triangleq\left\{e \in \mathbb{R}:|e| \leq \delta_{\theta}\right\} \\
& e_{\phi} \in \mathcal{E}_{\phi} \triangleq\left\{e \in \mathbb{R}:|e| \leq \delta_{\phi}\right\}
\end{aligned}
$$

with $\delta_{\theta}$ and $\delta_{\phi}$ denoting the maximum possible angular deviations. Then, the robust counterpart of (21) in the case of orientation uncertainties can be stated as

$$
\underset{\mathbf{p}}{\operatorname{minimize}} \max _{\substack{e_{\theta} \in \mathcal{E}_{\theta} \\ e_{\phi} \in \mathcal{E}_{\phi}}} \operatorname{trace}\left\{\left(\left(\mathbf{I}_{3} \otimes \mathbf{p}\right)^{T} \boldsymbol{\Gamma}\left(\boldsymbol{n}_{\mathrm{r}}\left(\hat{\theta}-e_{\theta}, \hat{\phi}-e_{\phi}\right)\right)\right)^{-1}\right\}
$$$$
\text { subject to } \mathbf{p} \in \mathcal{P}
$$

where $\boldsymbol{n}_{\mathrm{r}}(\cdot, \cdot)$ is as defined in (46) and $\boldsymbol{\Gamma}\left(\boldsymbol{n}_{\mathrm{r}}(\theta, \phi)\right)$ is the matrix $\boldsymbol{\Gamma}$ in (10) evaluated at $\boldsymbol{n}_{\mathrm{r}}=\boldsymbol{n}_{\mathrm{r}}(\theta, \phi)$.

\section{Iterative Entropic Regularization Algorithm}

In this part, we develop a unified power allocation algorithm design for solving the robust optimization problems in (45) and (49). To this end, let the error vectors and the corresponding uncertainty sets in (44) and (48) be defined as follows:

$$
\widetilde{\mathbf{e}} \triangleq \begin{cases}\mathbf{e}_{l_{\mathrm{r}}}, & \text { uncertainty in } \mathrm{VLC} \text { receiver location } \\ \left(e_{\theta}, e_{\phi}\right), & \text { uncertainty in VLC receiver orientation }\end{cases}
$$

$$
\widetilde{\mathcal{E}} \triangleq \begin{cases}\mathcal{E}_{l_{\mathrm{r}}}, & \text { uncertainty in VLC receiver location } \\ \mathcal{E}_{\theta} \times \mathcal{E}_{\phi}, & \text { uncertainty in VLC receiver orientation }\end{cases}
$$

In addition, the objective functions in (45) and (49) can be represented by

$\psi(\mathbf{p}, \widetilde{\mathbf{e}}) \triangleq\left\{\begin{array}{l}\operatorname{trace}\left\{\left(\left(\mathbf{I}_{3} \otimes \mathbf{p}\right)^{T} \boldsymbol{\Gamma}\left(\hat{\boldsymbol{l}}_{\mathrm{r}}-\mathbf{e}_{\boldsymbol{l}_{\mathrm{r}}}\right)\right)^{-1}\right\} \\ \operatorname{trace}\left\{\left(\left(\mathbf{I}_{3} \otimes \mathbf{p}\right)^{T} \boldsymbol{\Gamma}\left(\boldsymbol{n}_{\mathrm{r}}\left(\hat{\theta}-e_{\theta}, \hat{\phi}-e_{\phi}\right)\right)\right)^{-1}\right\}\end{array}\right.$ 
where the first and second rows denote, respectively, the cases for the uncertainty in the location and the orientation. Then, based on (50)-(52), the problems in (45) and (49) can be unified into a single optimization framework as follows:

$$
\underset{\mathbf{p}}{\operatorname{minimize}} \max _{\widetilde{\mathbf{e}} \in \widetilde{\mathcal{E}}} \psi(\mathbf{p}, \widetilde{\mathbf{e}}) \quad \text { subject to } \mathbf{p} \in \mathcal{P}
$$

The inner problem in (53) is not convex since $\psi(\mathbf{p}, \widetilde{\mathbf{e}})$ is

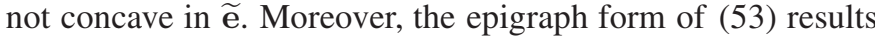
in a semi-infinite optimization problem whose constraints (in the form of $\psi(\mathbf{p}, \widetilde{\mathbf{e}}) \leq t, \forall \widetilde{\mathbf{e}} \in \widetilde{\mathcal{E}}$, for some $t \in \mathbb{R})$ do not admit a tractable convex reformulation, as in (37b). Furthermore, the exhaustive search method for solving (53) has a computational complexity that is exponential in the number of LED transmitters $N_{\mathrm{L}}$. Therefore, it is challenging to solve (53) in a computationally efficient manner via conventional techniques.

To tackle the robust design problem in (53), our algorithmic approach is to use an iterative entropic regularization procedure that successively decreases the objective value of the outer problem by updating the power vector $\mathbf{p}$ while simultaneously refining the optimal value of the inner maximization problem [71], [72]. Let the objective function of the outer problem in (53) be defined as

$$
\Psi(\mathbf{p}) \triangleq \max _{\widetilde{\mathbf{e}} \in \widetilde{\mathcal{E}}} \psi(\mathbf{p}, \widetilde{\mathbf{e}}) .
$$

The continuous uncertainty set $\widetilde{\mathcal{E}}$ can be discretized using $n$ points in $\widetilde{\mathcal{E}}$ to obtain a subset $\widetilde{\mathcal{E}}_{n}$ of $\widetilde{\mathcal{E}}$. Then, $\Psi(\mathbf{p})$ in (54) can be approximated by $\Psi^{n}(\mathbf{p}) \triangleq \max _{\widetilde{\mathbf{e}} \in \overline{\mathcal{E}}_{n}} \psi(\mathbf{p}, \widetilde{\mathbf{e}})$. To circumvent the non-differentiability of $\Psi^{n}(\mathbf{p})$, we can employ the following entropic regularized/smoothed approximation of the max function [71], [58, p. 72]:

$$
\Psi_{\varrho}^{n}(\mathbf{p}) \triangleq \frac{1}{\varrho} \log \left\{\sum_{\widetilde{\mathbf{e}} \in \widetilde{\mathcal{E}}_{n}} \exp (\varrho \psi(\mathbf{p}, \widetilde{\mathbf{e}}))\right\}
$$

where $\varrho$ is the regularization constant [72].

Based on the regularized function in (55), we propose the iterative entropic regularization algorithm in Algorithm 1, which consists of the following steps [71], [72]:

- Outer Minimization: The objective function $\Psi(\mathbf{p})$ in (54) is approximated by the smoothed version $\Psi_{\varrho}^{n}(\mathbf{p})$ in (55). The resulting convex problem ${ }^{10}$ in (56) can efficiently be solved via standard tools of convex optimization [58].

- Inner Maximization: Using the power vector $\mathbf{p}^{\star}$ obtained from the outer minimization step, the inner maximization problem of (53) is solved in (57) by performing a three (two)-dimensional grid search over $\mathcal{E}$ for the case of the uncertainty in the location (orientation) of the VLC receiver.

Algorithm 1 can be shown to converge to a global minimum of (53) [71]. It is worth noting that the computational burden of (53) is significantly reduced via Algorithm 1 as compared

\footnotetext{
${ }^{10}$ Since $\psi(\mathbf{p}, \widetilde{\mathbf{e}})$ is a convex function of $\mathbf{p}$ for a given $\widetilde{\mathbf{e}}$ (see (52) and Lemma 1) and the log-sum-exp function is convex [58, p. 72], the resulting composition $\Psi_{\varrho}^{n}(\mathbf{p})$ is convex in $\mathbf{p}$.
}

Algorithm 1 Iterative Entropic Regularization Algorithm to Solve the Robust Power Allocation Problem in (53)

\section{Initialization:}

Select $\widetilde{\mathbf{e}}_{1} \in \widetilde{\mathcal{E}}$, set $\widetilde{\mathcal{E}}_{1}=\left\{\widetilde{\mathbf{e}}_{1}\right\}, n=1$ and $k=1$.

Select $\varrho>0, \epsilon \in(0,1), \varsigma>0$ and $N_{\text {grid }} \in \mathbb{Z}^{+}$.

\section{Iterative Step:}

(Outer Problem) Solve the following convex optimization problem with a tolerance level of $\epsilon^{k}$ :

$$
\mathbf{p}^{\star}=\arg \min _{\mathbf{p} \in \mathcal{P}} \Psi_{\varrho}^{n}(\mathbf{p})
$$

where $\Psi_{\varrho}^{n}(\mathbf{p})$ is given by (55).

(Inner Problem) Obtain a new candidate from the uncertainty region $\widetilde{\mathcal{E}}$ using a grid search over the prespecified $N_{\text {grid }}$ points:

$$
\widetilde{\mathbf{e}}_{n+1}=\arg \max _{\widetilde{\mathbf{e}} \in \widetilde{\mathcal{E}}} \psi\left(\mathbf{p}^{\star}, \widetilde{\mathbf{e}}\right)
$$

where $\psi(\mathbf{p}, \widetilde{\mathbf{e}})$ is as defined in (52).

Update $k=k+1$.

(Check the Objective Value)

if $\psi\left(\mathbf{p}^{\star}, \widetilde{\mathbf{e}}_{n+1}\right)>\Psi_{\varrho}^{n}\left(\mathbf{p}^{\star}\right)$ then

Set $\widetilde{\mathcal{E}}_{n+1}=\widetilde{\mathcal{E}}_{n} \cup\left\{\widetilde{\mathbf{e}}_{n+1}\right\}$.

Update $n=n+1$.

Update $\varrho=\max \left(\varrho, \log (n)^{2}\right)$.

\section{end if}

(Check the Tolerance Value)

if $\epsilon^{k}+\log (n) / \varrho>\varsigma$ then

Update $\varrho=\varrho+\log (n)$.

\section{end if}

\section{Stopping Criteria:}

$\psi\left(\mathbf{p}^{\star}, \widetilde{\mathbf{e}}_{n+1}\right) \leq \Psi_{\varrho}^{n}\left(\mathbf{p}^{\star}\right)$ and $\epsilon^{k}+\log (n) / \varrho \leq \varsigma$.

to the exhaustive search approach, as mentioned in the next subsection.

\section{Complexity Analysis}

In this part, we discuss the computational complexity of Algorithm 1 and compare it with that of the exhaustive search based solution of (53). At each iteration, Algorithm 1 involves solving a convex optimization problem and a grid search over the uncertainty region. Let $\mathcal{O}(C)$ denote the complexity of the convex optimization problem in (56) and $N_{\text {grid }}$ the number of points employed for the grid search over $\widetilde{\mathcal{E}}$ in (57). Then, the per-iteration complexity of Algorithm 1 is given by $\mathcal{O}(C)+\mathcal{O}\left(N_{\text {grid }}\right)$. Regarding the exhaustive search technique for solving (53), let each axis of the feasible region $\mathcal{P} \subset \mathbb{R}^{N_{\mathrm{L}}}$ be discretized using $\mathcal{O}(M)$ different values. Thus, the outer iteration of (53) has a computational complexity of $\mathcal{O}\left(M^{N_{\mathrm{L}}}\right)$. Utilizing $N_{\text {grid }}$ points for the inner iteration, the overall complexity becomes $\mathcal{O}\left(M^{N_{\mathrm{L}}} N_{\text {grid }}\right)$. Therefore, the complexity of the exhaustive search method grows exponentially with the number of LED transmitters, whereas that of Algorithm 1 is primarily determined by the convex problem in (56), which can be solved in polynomial time [65]. As a result, Algorithm 1 has significantly lower computational complexity than the exhaustive search based solution. 


\section{Minimum Power Consumption Problem}

In practical indoor VLP systems, the power consumption of LEDs and the localization error of VLC receivers must be jointly considered in a power optimization problem. In Section III, Section IV and Section V, the aim is to minimize the localization error while satisfying power and illumination related constraints. However, for improved energy efficiency of VLP systems, the total power consumption of LEDs must also be taken into account in addition to localization performance requirements [73]. ${ }^{11}$ Therefore, similar to the minimal illumination level problem in VLC systems [18], [54], we consider the minimum power consumption problem for VLP systems, where the objective is to minimize the total power consumption of LEDs while keeping the CRLB of the VLC receiver below a predefined level. In the following subsections, we first investigate the problem of total power minimization under perfect knowledge of localization parameters and then study robust power allocation designs in the presence of uncertainties.

\section{A. Power Minimization With Perfect Knowledge}

In the absence of uncertainties in localization parameters, the minimum power consumption problem can be formulated as follows:

$$
\begin{array}{ll}
\underset{\mathbf{p}}{\operatorname{minimize}} & \mathbf{1}^{T} \mathbf{p} \\
\text { subject to } & \operatorname{trace}\left\{\mathbf{J}^{-1}(\mathbf{p})\right\} \leq \varepsilon \\
& \mathbf{p} \in \mathcal{P}_{s}
\end{array}
$$

where $1^{T} \mathbf{p}$ determines the total electrical power consumption, $\mathcal{P}_{s} \triangleq \mathcal{P}_{1} \cap \mathcal{P}_{3} \cap \mathcal{P}_{4}$ and $\varepsilon$ represents the maximum tolerable CRLB level for the localization of the VLC receiver. In (58), we seek to find the most energy-efficient LED power assignment scheme satisfying a certain level of localization accuracy. As implied by Lemma 1, the optimization problem in (58) is convex.

The significance of the considered problem in (58) for VLP systems lies in the fact that it yields the minimum value of $P_{\mathrm{T}}$ in (13), above which the optimal solution of (21) always achieves a lower CRLB than the specified design level, $\varepsilon$. In other words, a certain level of localization performance is guaranteed by setting $P_{\mathrm{T}}$ above the obtained minimum value in (58), as in the minimal illumination level problem in VLC systems [54].

\section{B. Robust Power Minimization With Imperfect Knowledge}

In this part, we consider the robust counterpart of the power minimization problem in (58) under deterministic normbounded uncertainty in matrix $\Gamma$ in (10) based on the error model in (26). Thus, we assume that the errors in $\Gamma$ belong to a bounded uncertainty region as in Section IV-A and develop a robust approach that guarantees the localization performance

\footnotetext{
${ }^{11}$ Energy efficient localization algorithms have been considered also for RF sensor networks in the literature (e.g., [74]).
}

\begin{tabular}{|c|c|}
\hline $\begin{array}{l}\text { Location of LED-1, } \boldsymbol{l}_{\mathrm{t}}^{1} \\
\text { Location of LED-2, } \boldsymbol{l}_{\mathrm{t}}^{2} \\
\text { Location of LED-3, } \boldsymbol{l}_{\mathrm{t}}^{3} \\
\text { Location of LED-4, } \boldsymbol{l}_{\mathrm{t}}^{4} \\
\text { Orientation of LEDs, } \boldsymbol{n}_{\mathrm{t}}^{i}(i=1,2,3,4)\end{array}$ & $\begin{array}{l}{\left[\begin{array}{lll}1 & 1 & 5\end{array}\right]^{T} \mathrm{~m}} \\
{\left[\begin{array}{lll}1 & 9 & 5\end{array}\right]^{T} \mathrm{~m}} \\
{\left[\begin{array}{lll}9 & 1 & 5\end{array}\right]^{T} \mathrm{~m}} \\
{\left[\begin{array}{lll}9 & 9 & 5\end{array}\right]^{T} \mathrm{~m}} \\
{\left[\begin{array}{lll}0 & 0 & -\end{array}\right]^{T}}\end{array}$ \\
\hline $\begin{array}{l}\text { Location of VLC Receiver, } \boldsymbol{l}_{\mathrm{r}} \\
\text { Orientation of VLC Receiver, } \boldsymbol{n}_{\mathrm{r}}\end{array}$ & $\begin{array}{l}{\left[\begin{array}{llll}3 & 3 & 0.5\end{array}\right]^{T} \mathrm{~m}} \\
{\left[\begin{array}{llll}0.5 & 0 & 0.866\end{array}\right]^{T}}\end{array}$ \\
\hline Location of Illumination Constraint- $1, \mathbf{x}_{1}$ & {$\left[\begin{array}{lll}1 & 1 & 1\end{array}\right]^{T} \mathrm{~m}$} \\
\hline Location of Illumination Constraint- $2, \mathbf{x}_{2}$ & {$\left[\begin{array}{lll}1 & 9 & 1\end{array}\right]^{T} \mathrm{~m}$} \\
\hline Location of Illumination Constraint- $3, \mathbf{x}_{3}$ & {$\left[\begin{array}{lll}9 & 1 & 1\end{array}\right]^{T} \mathrm{~m}$} \\
\hline Location of Illumination Constraint- $4, \mathbf{x}_{4}$ & {$\left[\begin{array}{lll}9 & 9 & 1\end{array}\right]^{T} \mathrm{~m}$} \\
\hline
\end{tabular}

TABLE I

LOCATIONS AND ORIENTATIONS

measure for all the uncertainties in the specified region. Accordingly, the robust design problem can be formulated as

$$
\begin{aligned}
& \underset{\mathbf{p}}{\operatorname{minimize}} \mathbf{1}^{T} \mathbf{p} \\
& \text { subject to } \operatorname{trace}\left\{\left(\left(\mathbf{I}_{3} \otimes \mathbf{p}\right)^{T}(\widehat{\boldsymbol{\Gamma}}-\boldsymbol{\Delta} \boldsymbol{\Gamma})\right)^{-1}\right\} \leq \varepsilon, \\
& \forall \boldsymbol{p} \boldsymbol{\Gamma} \in \mathcal{E} \\
& \mathbf{p} \in \mathcal{P}_{s}
\end{aligned}
$$

where $\mathcal{E}$ is given by (27) and $\varepsilon$ represents the constraint on the worst-case CRLB. Similar to (28), the semi-infinite programming problem in (59) can equivalently be reformulated as a convex problem, as shown in the following proposition.

Proposition 2: The robust power allocation problem in (59) is equivalent to the following convex optimization problem:

$$
\begin{aligned}
\underset{\mathbf{p}, \mathbf{H}, s, \mu}{\operatorname{minimize}} & \mathbf{1}^{T} \mathbf{p} \\
\text { subject to } & \operatorname{trace}\{\mathbf{H}\} \leq \varepsilon-d s \\
& \mathbf{\Phi}(\mathbf{p}, \mathbf{H}, s, \mu) \succeq 0 \\
& \mathbf{H} \succeq 0, \mu \geq 0, \mathbf{p} \in \mathcal{P}_{s}
\end{aligned}
$$

where $\mathbf{\Phi}(\mathbf{p}, \mathbf{H}, s, \mu)$ is defined as in (33).

Proof: The proof directly follows from that of Proposition 1.

\section{NumERICAL Results}

In this section, we provide numerical examples to investigate the performance of the proposed optimal and robust power allocation designs for VLP systems.

\section{A. Simulation Setup}

We consider a VLP scenario in a room of size $10 \times$ $10 \times 5 \mathrm{~m}^{3}$, where there exist $N_{\mathrm{L}}=4$ LED transmitters and a VLC receiver. The locations and the orientations of the LED transmitters and the VLC receiver are provided in Table I. In addition, $L=4$ locations are determined for individual illumination constraints, which are also displayed in Table I. The average illuminance in (18) is calculated over the horizontal plane of the room at a fixed height of $1 \mathrm{~m}$.

The scaled version of the transmitted signal, $\widetilde{s}_{i}(t)$, in (4) is modeled as $\widetilde{s}_{i}(t)=\frac{2}{3}\left(1-\cos \left(2 \pi t / T_{s, i}\right)\right)\left(1+\cos \left(2 \pi f_{c, i} t\right)\right)$ for $i=1, \ldots, N_{\mathrm{L}}$ and $t \in\left[0, T_{s, i}\right]$, where $T_{s, i}$ is the pulse 
TABLE II

SIMULATION PARAMETERS

\begin{tabular}{|l|l|}
\hline Responsivity of Photo Detector, $R_{p}$ & $0.4 \mathrm{~mA} / \mathrm{mW}$ \\
Area of Photo Detector, $S$ & $1 \mathrm{~cm}^{2}$ \\
Spectral Density Level of Noise, $\sigma^{2}$ & $1.3381 \times 10^{-22} \mathrm{~W} / \mathrm{Hz}$ \\
LED Lambertian Order, $m_{i}(i=1,2,3,4)$ & 1 \\
LED Luminous Efficacy, $\kappa_{i}(i=1,2,3,4)$ & $284 \mathrm{~lm} / \mathrm{W}$ \\
Min. LED Optical Power & $5 \mathrm{~W}$ \\
Max. LED Optical Power & $20 \mathrm{~W}$ \\
Min. Illuminance Level, $\widetilde{\mathcal{I}}_{\text {avg }}, \widetilde{\mathcal{I}}_{\ell}(\ell=1,2,3,4)$ & $30 \mathrm{~lx}$ \\
Transmitted Pulse Width, $T_{s, i}(i=1,2,3,4)$ & $1 \mu \mathrm{s}$ \\
Center Frequency, $f_{c, i}(i=1,2,3,4)$ & $40+20(i-1) \mathrm{MHz}$ \\
\hline
\end{tabular}

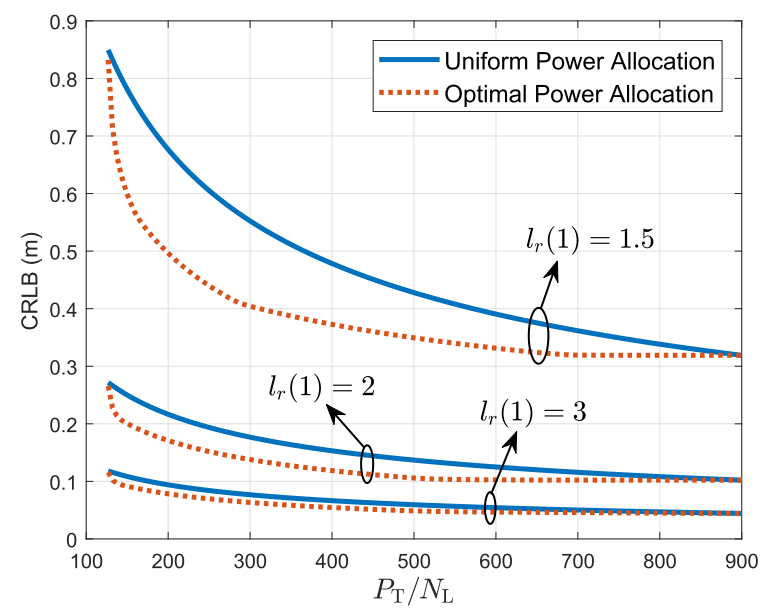

Fig. 1. CRLB of (21) versus $P_{\mathrm{T}} / N_{\mathrm{L}}$ for optimal and uniform power allocation strategies for various locations of the VLC receiver.

width and $f_{c, i}$ is the center frequency [45], [75]. ${ }^{12}$ From (6), the optical power of $\widetilde{s}_{i}(t)$ is calculated as $\widetilde{E}_{i}^{\text {opt }}=2 / 3$. In accordance with [41], [43], [45], [64], [75], the VLP system parameters utilized throughout the simulations are given in Table II. In addition, an asynchronous VLP system is considered, i.e., the time offsets $\left\{\Delta_{i}\right\}_{i=1}^{N_{\mathrm{L}}}$ in (2) are modeled as unknown parameters.

\section{B. Power Allocation With Perfect Knowledge}

In this part, we investigate the effects of the proposed optimal power allocation approach on the localization performance of the VLC receiver under the assumption of perfect knowledge of localization related parameters. Since this is the first study to consider power allocation in VLP systems, the uniform power allocation strategy (i.e., $P_{i}=P_{\mathrm{T}} / N_{\mathrm{L}}$, $i=1, \ldots, N_{\mathrm{L}}$ ) is also illustrated for comparison purposes.

Fig. 1 plots the CRLB achieved by the optimal solution of (21) versus $P_{\mathrm{T}} / N_{\mathrm{L}}$, which determines the average electrical power limit, for various locations of the VLC receiver. It is observed that the optimal power allocation approach can provide significant improvements in localization performance over the conventional uniform power allocation approach. In addition, we note that the performance improvement becomes more pronounced as the VLC receiver moves

\footnotetext{
${ }^{12}$ The constant factor $2 / 3$ is included to satisfy $\int_{0}^{T_{s, i}}\left(\widetilde{s}_{i}(t)\right)^{2} d t / T_{s, i}=1$, as indicated in Section III-A.
}

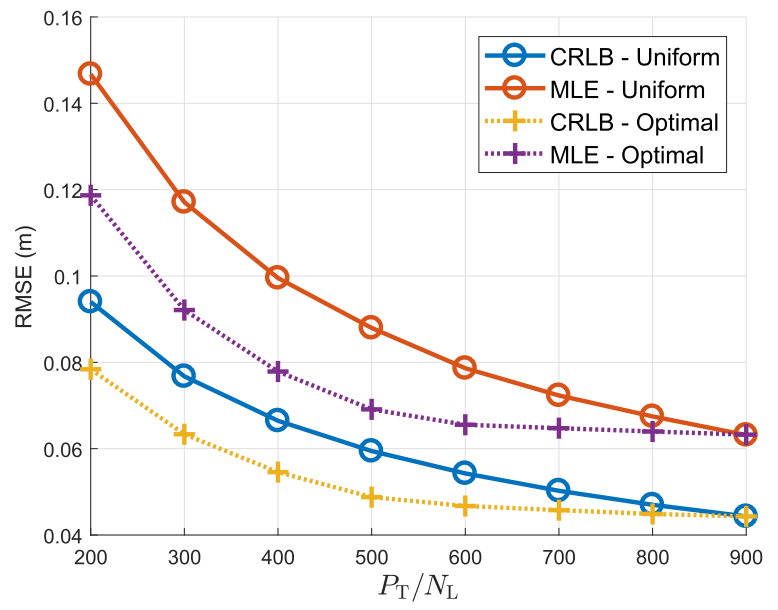

Fig. 2. RMSEs of the ML estimators and the CRLBs corresponding to optimal and uniform power allocation strategies with respect to $P_{\mathrm{T}} / N_{\mathrm{L}}$.

away from the center of the room. The reason is that the contribution of each LED to the Fisher information in (8) becomes almost equal at the room center whereas the LEDs are less symmetric at the corners. Moreover, due to the limited linear operation regime of the LEDs, the optimal strategy exhibits a similar performance to that of the uniform strategy for sufficiently high values of $P_{\mathrm{T}}$. Furthermore, when $P_{\mathrm{T}}$ is lower than a certain value, the problem becomes infeasible due to the average illumination constraint, and the uniform and optimal strategies achieve the same CRLB at that value of $P_{\mathrm{T}}$.

In order to evaluate how the optimization based on the CRLB metric reduces the true positioning error, we implement the ML estimator in [49, Eq. (44)] using the LED optical powers obtained from the optimal solution of (21). Fig. 2 shows the root-MSEs (RMSEs) of the ML estimators corresponding to the optimal and uniform power allocation strategies along with the corresponding CRLBs with respect to $P_{\mathrm{T}} / N_{\mathrm{L}}$. We observe that power optimization based on the CRLB metric can provide a level of performance enhancement in terms of the RMSE of the ML estimator that is congruent with what is predicted by the CRLB.

To investigate the power allocation performance in the presence of multiple VLC receivers, we also consider the problem of average CRLB minimization of $N_{\mathrm{V}}$ receivers

$$
\begin{aligned}
& \underset{\mathbf{p}}{\operatorname{minimize}} \frac{1}{N_{\mathrm{V}}} \sum_{\ell=1}^{N_{\mathrm{V}}} \operatorname{trace}\left\{\mathbf{J}_{\ell}^{-1}(\mathbf{p})\right\} \\
& \text { subject to } \mathbf{p} \in \mathcal{P}
\end{aligned}
$$

where $\mathbf{J}_{\ell}(\mathbf{p})$ denotes the FIM for the $\ell$ th receiver. Fig. 3 depicts the average CRLB performance of (61) with three VLC receivers against $P_{\mathrm{T}} / N_{\mathrm{L}}$ for the cases of optimal and uniform power allocation. Similar to the scenario with a single VLC receiver, substantial localization performance gains within the dynamic range of the LEDs can be achieved via power optimization with multiple receivers. Hence, different receivers can simultaneously utilize the available power 


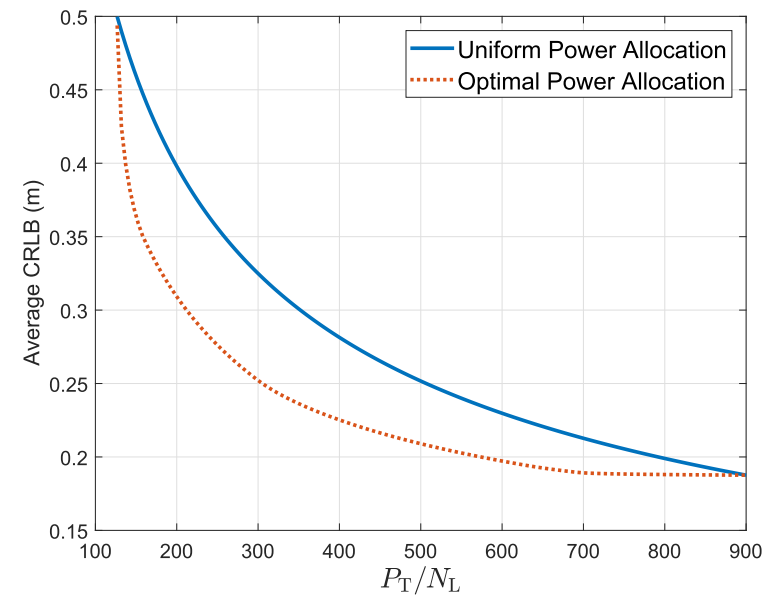

Fig. 3. Average CRLB of three VLC receivers in (61) versus $P_{\mathrm{T}} / N_{\mathrm{L}}$ for optimal and uniform power allocation strategies, where the receiver locations are given by $\boldsymbol{l}_{\mathrm{r}}^{1}=\left[\begin{array}{lll}1.5 & 3 & 0.5\end{array}\right]^{T} \mathrm{~m}, \boldsymbol{l}_{\mathrm{r}}^{2}=\left[\begin{array}{lll}3 & 3 & 0.5\end{array}\right]^{T} \mathrm{~m}, \boldsymbol{l}_{\mathrm{r}}^{3}=\left[\begin{array}{lll}6 & 8 & 0.5\end{array}\right]^{T} \mathrm{~m}$, and the receiver orientations are given by $\boldsymbol{n}_{\mathrm{r}}^{1}=\left[\begin{array}{lll}0.5 & 0.866\end{array}\right]^{T}, \boldsymbol{n}_{\mathrm{r}}^{2}=$ $\left[\begin{array}{lll}0.5 & 0 & 0.866\end{array}\right]^{T}, \boldsymbol{n}_{\mathrm{r}}^{3}=\left[\begin{array}{llll}-0.2198 & -0.6040 & 0.7660\end{array}\right]^{T}$.

resources at the LED transmitters, resulting in improved efficiency of the VLP system.

\section{Robust Power Allocation in the Presence of Overall System Uncertainty}

To illustrate the performance of the robust power allocation in the presence of overall system uncertainty, as discussed in Section IV, several numerical examples are provided for the problem in (32), which is equivalent to the original robust problem in (28). Since the goal of robustness is to optimize the worst-case performance, we investigate the worst-case CRLBs achieved by the following strategies:

- Robust: The robust strategy takes into account the uncertainty in $\boldsymbol{\Gamma}$ and solves the problem in (32). Then, the resulting optimal value $t^{\star}$ of the slack variable $t$ yields the worst-case CRLB.

- Non-robust: The non-robust strategy ignores the uncertainty in $\Gamma$ and directly utilizes the nominal matrix $\widehat{\Gamma}$ in (26) to solve the power allocation problem in (21). To obtain the worst-case CRLB corresponding to optimal power vector $\mathbf{p}^{\mathrm{n}-\mathrm{rob}}$ of (21), $\mathbf{p}^{\mathrm{n}-\mathrm{rob}}$ is inserted into (32) as a fixed quantity. Hence, the worst-case CRLB $t^{\star}$ is calculated by solving

$$
\begin{aligned}
t^{\star}=\min _{t, \mathbf{H}, s, \mu} t & \\
\text { subject to } & \operatorname{trace}\{\mathbf{H}\} \leq t-d s \\
& \mathbf{\Phi}\left(\mathbf{p}^{\mathrm{n}-\mathrm{rob}}, \mathbf{H}, s, \mu\right) \succeq 0 \\
& \mathbf{H} \succeq 0 \\
& \mu \geq 0
\end{aligned}
$$

where $\mathbf{\Phi}(\mathbf{p}, \mathbf{H}, s, \mu)$ is given by (33).

- Uniform: In this strategy, the uniform power allocation vector is used and the corresponding worst-case CRLB is obtained via (62) by replacing $\mathbf{p}^{\mathrm{n}-\mathrm{rob}}$ with the uniform power vector.

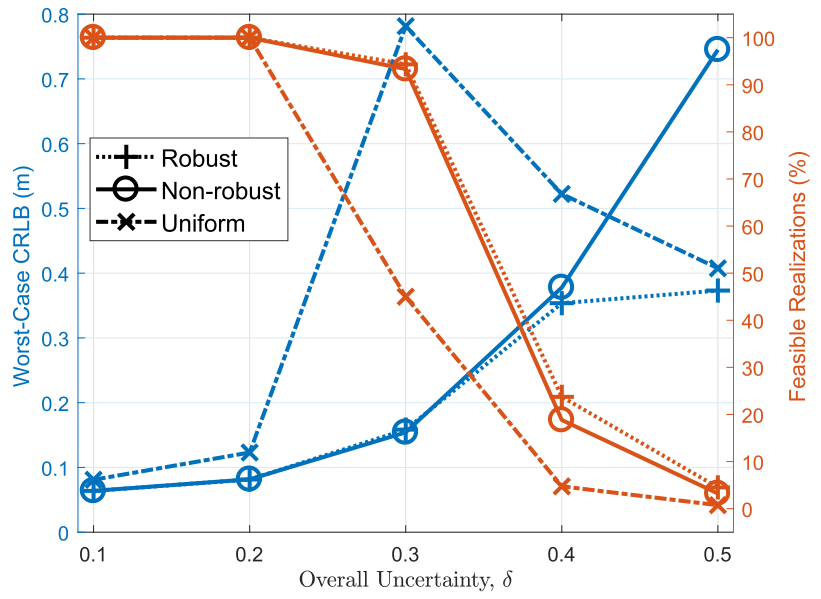

Fig. 4. Worst-case CRLB and the percentage of feasible realizations in (28) versus the level of uncertainty $\delta$, where the average power limit is $P_{\mathrm{T}} / N_{\mathrm{L}}=400$.

The worst-case CRLBs are averaged over 100 Monte Carlo realizations. For each realization, an error matrix $\Delta \Gamma$ is randomly chosen from the uncertainty set $\mathcal{E}$ in (27) and the nominal matrix $\widehat{\Gamma}$ is generated according to (26). Then, each strategy is evaluated by using realizations for which that strategy is feasible. ${ }^{13}$

Fig. 4 shows the worst-case CRLB performance and the feasibility rate of the considered power allocation strategies against the level of uncertainty $\delta$ in (27). It is observed that the performance of all the strategies deteriorates as the uncertainty increases, as expected. For small uncertainty regions (i.e., small $\delta$ ), the robust strategy has almost the same performance as its non-robust counterpart. However, the robust strategy outperforms the non-robust strategy for large uncertainty regions, which results from the design philosophy in (28). More specifically, since the nominal matrix $\widehat{\Gamma}$ deviates substantially from the true matrix $\boldsymbol{\Gamma}$ for large values of $\delta$, the non-robust strategy, which treats $\widehat{\Gamma}$ as the true matrix in LED power optimization, results in poor worst-case localization performance. On the other hand, the robust approach attempts to minimize the performance degradation by utilizing the properties of the uncertainty region $\mathcal{E}$ in (27).

As noted from Fig. 4, the robust strategy also provides the highest feasibility rate among all the strategies since the feasible region of (62) is smaller than that of (32) (the constraint set (32e) is replaced by a single point in (62)). In addition, the feasibility rate of the uniform strategy undergoes a sharp decline after a certain level of uncertainty, which distorts the monotonic behavior of its worst-case CRLB around the point where this decline occurs. It is worth noting that the non-robust strategy achieves a higher feasibility rate and lower worst-case CRLB than the uniform strategy for small

\footnotetext{
${ }^{13}$ More specifically, we fix the number of feasible realizations beforehand and continue to pick new matrices from the uncertainty region until the predefined number of feasible realizations is reached. For the robust strategy, feasibility refers to the problem in (32) being feasible for a given realization $\widehat{\boldsymbol{\Gamma}}$. For the non-robust and uniform strategies, feasibility means that the problem in (62) is feasible, which is equivalent to the worst-case CRLB in (62a) being finite.
} 
$\delta$, but this trend changes as $\delta$ increases. The reason is that for small $\delta$, the non-robust approach can find near-optimal power allocation vectors in the sense of (28) (since solving (21) is almost equivalent to solving (28) for small $\delta$ ) whereas the uniform power vector does not take into account the localization related parameters (e.g., locations and orientations of the LED transmitters and the VLC receiver) and assigns equal power to all the LEDs, which leads to low feasibility rates and large errors in localization. On the other hand, for high $\delta$, the performance of the non-robust strategy becomes worse than that of the uniform strategy with increasing errors in $\widehat{\Gamma}$.

\section{Robust Power Allocation in the Presence of Individual Parameter Uncertainties}

In this part, we consider the robust power allocation schemes designed for the case of individual parameter uncertainties, as discussed in Section V. In the simulations, we explore the performance of the three strategies as mentioned in Section VII-C using 100 Monte Carlo realizations. The robust strategy is obtained by solving (53) via Algorithm $1 .{ }^{14}$ For the non-robust strategy, the uncertainty set $\widetilde{\mathcal{E}}$ in (51) is ignored and the nominal parameters (i.e., $\hat{l}_{\mathrm{r}}$ in (43) or $(\hat{\theta}, \hat{\phi})$ in (47)) are employed for power allocation via (21). To compute the worst-case CRLB for a given power vector $\mathbf{p}^{*}$, which corresponds to $\Psi\left(\mathbf{p}^{*}\right)$ in (54), we use a multi-start optimization algorithm for globally solving the maximization problem in (54).

Fig. 5 depicts the worst-case CRLB performance versus the level of uncertainty in the VLC receiver location, $\delta_{l_{\mathrm{r}}}$, for the considered strategies. ${ }^{15}$ As seen from Fig. 5, the proposed robust power allocation approach always achieves lower worst-case CRLBs than the other two strategies. In addition, the performance benefit provided by the robust strategy over its non-robust counterpart becomes more evident for larger values of $\delta_{l_{\mathrm{r}}}$. Hence, the robust scheme in (45) can effectively exploit the characteristics of the uncertainty region $\mathcal{E}_{l_{\mathrm{r}}}$ in (44) to optimize the worst-case localization performance. This also indicates that the proposed power allocation algorithm in Algorithm 1 can successfully converge to the optimal solution of (45). Moreover, we observe that the uniform strategy

\footnotetext{
${ }^{14}$ For the grid search in (57) with the spherical uncertainty region in (44), the radial dimension is sampled uniformly using $N_{\text {rad }}=5$ points over $\left[0, \delta_{l_{\mathrm{r}}}\right]$, while the polar and azimuth dimensions are sampled uniformly using $N_{\text {orient }}=10$ points in the intervals $[0, \pi]$ and $[0,2 \pi]$, respectively. Hence, the grid search is executed over $N_{\text {grid }}=500$ discrete locations. Similarly, in the case of orientation uncertainty defined by the sets in (48a) and (48b), the polar $(\theta)$ and azimuth $(\phi)$ dimensions are discretized uniformly using $N_{\text {orient }}=10$ points over $\left[-\delta_{\theta}, \delta_{\theta}\right]$ and $\left[-\delta_{\phi}, \delta_{\phi}\right]$, respectively, which leads to a grid search over $N_{\text {grid }}=100$ points in the uncertainty region.

15 Even though the $x$-axis (uncertainty in location) and the $y$-axis (CRLB for location estimation) of Fig. 5 may seem to be conflicting with one another, they actually refer to different stages of a positioning/tracking system. In particular, the $x$-axis corresponds to the uncertainty in the receiver location that derives from the error covariance matrix of the tracking filter (e.g., Kalman filter) while the $y$-axis corresponds to the errors in location measurements, which can be quantified by the CRLB expression. In a VLP tracking scenario, the $y$-axis and the $x$-axis denote, respectively, the errors in the measurement and tracking blocks of a positioning system. Therefore, in Fig. 5, we aim to optimize the location measurement performance (i.e., CRLB) by utilizing the uncertainty information coming from the tracking block.
}

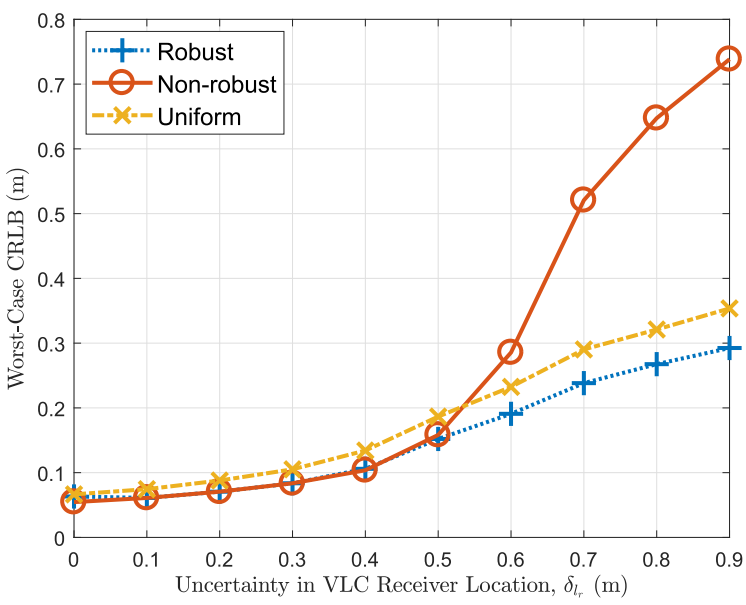

Fig. 5. Worst-case CRLB of (45) versus the level of uncertainty in the location of the VLC receiver $\delta_{l_{\mathrm{r}}}$, where the average power limit is $P_{\mathrm{T}} / N_{\mathrm{L}}=400$.

performs worse than the non-robust strategy for small $\delta_{l_{\mathrm{r}}}$. However, as $\delta_{l_{\mathrm{r}}}$ increases, the non-robust approach is outperformed by the uniform approach since the latter blindly assigns equal powers to the LEDs by disregarding parameter measurements whereas the former employs the highly inaccurate measurement of $\boldsymbol{l}_{\mathrm{r}}$ for power allocation of the LEDs.

In Fig. 6, we plot the worst-case CRLBs against the level of uncertainty in the polar angle of the VLC receiver $\left(\delta_{\theta}\right.$ in (48a)) for two different levels of uncertainty in the azimuth angle $\left(\delta_{\phi}\right.$ in $\left.(48 \mathrm{~b})\right)$. As seen from Fig. 6 , the proposed robust power allocation strategy offers the best worst-case CRLB performance among all strategies. In addition, we note that the performance gain achieved via the robust approach becomes more prominent for larger uncertainty regions $\mathcal{E}_{\theta}$ and $\mathcal{E}_{\phi}$ in (48). Moreover, additional numerical experiments for computing the worst-case RMSEs achieved by the considered power allocation approaches indicate that the level of improvement in the actual positioning error (i.e., RMSE) is analogous to that achieved by the worst-case CRLB optimization.

\section{E. Minimum Power Consumption Problem}

In this subsection, numerical examples are provided for the power allocation designs in Section VI.

1) Power Allocation With Perfect Knowledge: We explore the electrical power consumption corresponding to the optimal solution of (58) and provide a comparison with the uniform power allocation scheme, which is obtained from (58b) as

$$
P_{i}=\operatorname{trace}\left\{\left(\left(\mathbf{I}_{3} \otimes \mathbf{1}\right)^{T} \boldsymbol{\Gamma}\right)^{-1}\right\} / \varepsilon
$$

for $i=1, \ldots, N_{\mathrm{L}}$.

Fig. 7 plots $P_{\text {avg }}^{\star}$ versus $\sqrt{\varepsilon}$ for the optimal and uniform power allocation strategies, where $P_{\text {avg }}^{\star}$ corresponds to the optimal value of (58a) divided by $N_{\mathrm{L}}$ (which is proportional to the average electrical power consumption) and $\varepsilon$ is the desired CRLB level in (58b). From the figure, we observe power saving gains of around 30\% via the optimal approach for centimeter-level accuracy requirements. In addition, it is seen that the optimal strategy becomes equivalent to the uniform 


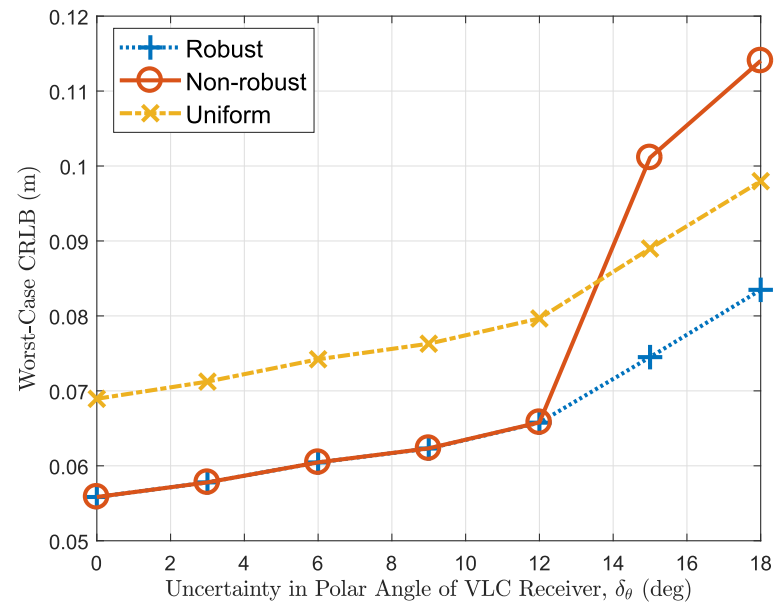

(a) $\delta_{\phi}=6^{\circ}$

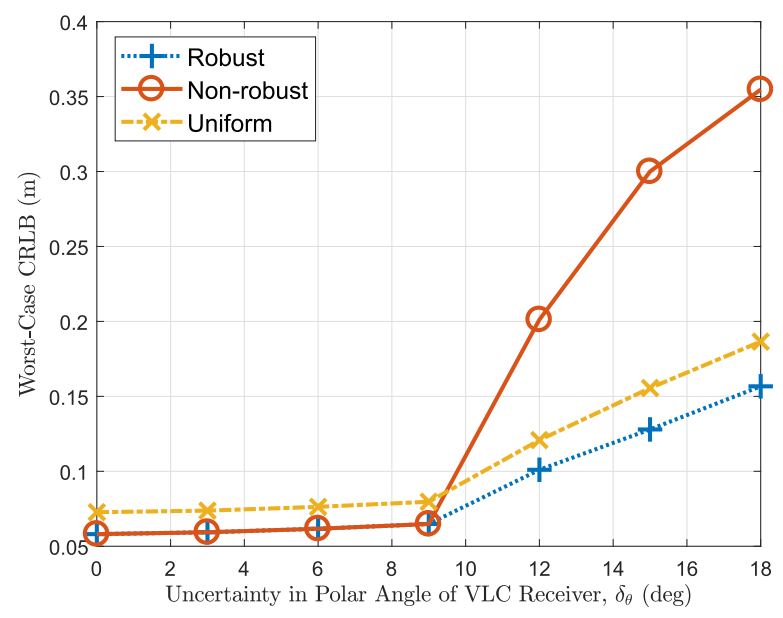

(b) $\delta_{\phi}=12^{\circ}$

Fig. 6. Worst-case CRLB of (49) versus the level of uncertainty in the polar angle of the VLC receiver $\delta_{\theta}$ for two different values of uncertainty in the azimuth angle $\delta_{\phi}$, where the average power limit is $P_{\mathrm{T}} / N_{\mathrm{L}}=400$.

strategy when the desired level of localization accuracy is sufficiently low, which results from the illumination constraints.

2) Robust Power Allocation With Imperfect Knowledge: We provide examples for the case of uncertainty in VLP system parameters, discussed in Section VI-B. For the robust strategy, we solve (60), which is equivalent to the original problem in (59) by Proposition 2, to get the optimal power vector, while the non-robust strategy is obtained by replacing $\widehat{\Gamma}$ with $\Gamma$ in (58). In addition, the uniform strategy is given by (63) with $\Gamma$ replaced by $\widehat{\Gamma}$.

Fig. 8 depicts the cumulative distribution function (CDF) of the CRLBs obtained by the considered strategies for two different uncertainty levels, $\delta=0.1$ and $\delta=0.2$, by setting the worst-case accuracy level as $\sqrt{\varepsilon}=0.1 \mathrm{~m}$. It is observed that the robust algorithm, which solves (60), satisfies the accuracy constraint in (59b) for all the realizations of $\boldsymbol{\Gamma}$ in accordance with the robust design approach, which also verifies the validity of Proposition 2. On the other hand, the non-robust and uniform strategies are not able to satisfy the accuracy constraint for approximately $50 \%$ of the realizations since they

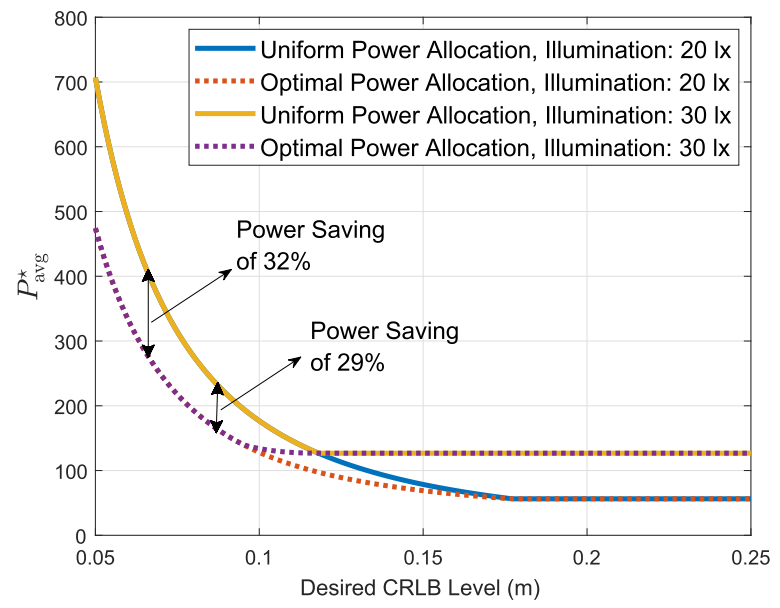

Fig. 7. Optimal value of (58a) divided by $N_{\mathrm{L}}\left(P_{\text {avg }}^{\star}\right)$ versus the desired CRLB level $\sqrt{\varepsilon}$ for optimal and uniform power allocation strategies under various illumination constraints.
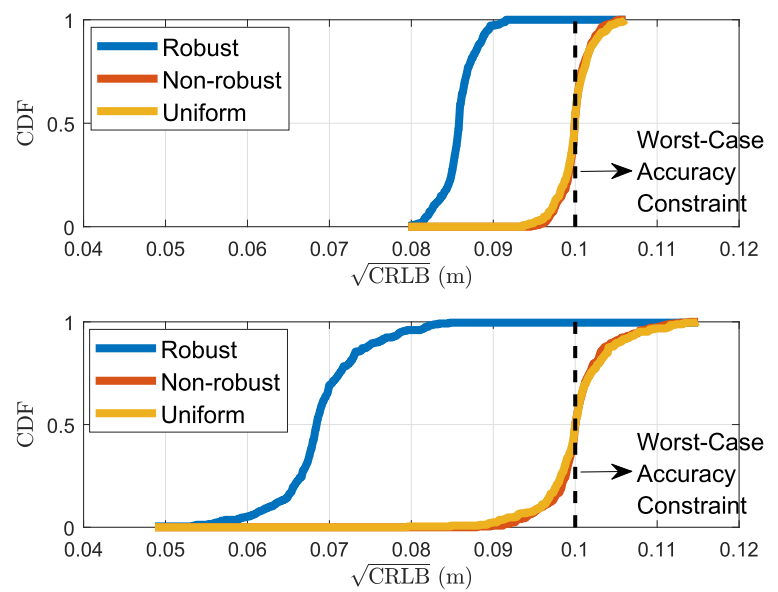

Fig. 8. CDF of localization CRLBs achieved by robust, non-robust and uniform strategies in the case of deterministic norm-bounded uncertainty for the matrix $\Gamma$, where the worst-case CRLB constraint in (59b) is set to $\sqrt{\varepsilon}=0.1 \mathrm{~m}$ and two different uncertainty levels are considered, namely, $\delta=0.1$ (above) and $\delta=0.2$ (below).

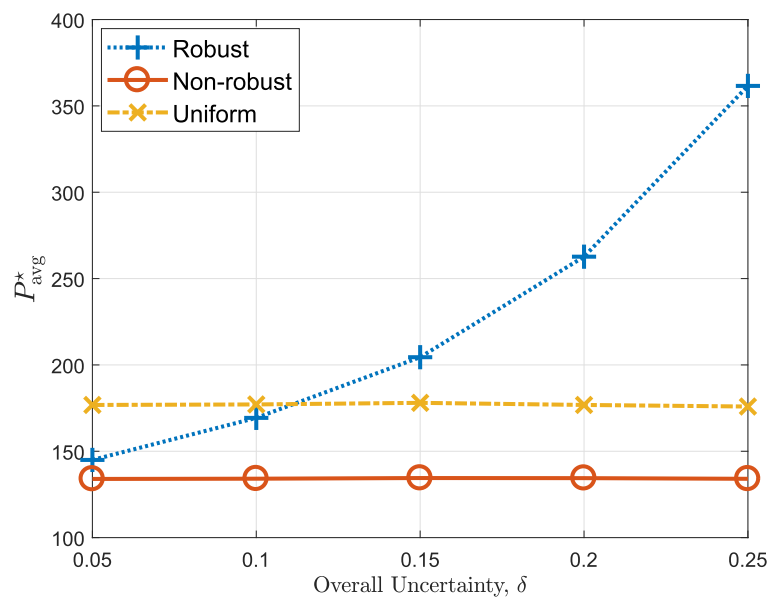

Fig. 9. Optimal value of (59a) divided by $N_{\mathrm{L}}\left(P_{\mathrm{avg}}^{\star}\right)$ versus the level of uncertainty $\delta$ for robust, non-robust and uniform power allocation strategies, where the worst-case accuracy constraint is $\sqrt{\varepsilon}=0.1 \mathrm{~m}$.

do not consider the uncertainty in $\boldsymbol{\Gamma}$ in allocating powers to the LEDs. Also, the CRLBs are observed to be more spread out for higher $\delta$ for all strategies. In Fig. 9, we show $P_{\text {avg }}^{\star}$ with 
respect to $\delta$, where $P_{\mathrm{avg}}^{\star}$ is the optimal value of (59a) divided by $N_{\mathrm{L}}$. It is seen that the robust strategy must utilize more transmission power with increasing $\delta$ in order to guarantee the specified level of accuracy for larger uncertainty regions, as expected. Hence, the relative performance gain of the robust strategy can be achieved at the cost of higher transmit powers and increased computational complexity, which results from solving (60) rather than the original problem (58). However, as opposed to the non-robust power allocation, the robust approach provides a solid theoretical guarantee for satisfying the worst-case CRLB constraint in (59b).

\section{CONCLUding REMARKS}

In this paper, we have considered the problem of optimal power allocation for LED transmitters in a VLP system. The optimization problem has been formulated to minimize the CRLB for the localization of the VLC receiver under practical constraints on transmission powers and illumination levels. Under the assumption of perfect knowledge of localization related parameters, the power allocation problem has been shown to be convex and thus efficiently solvable. In the presence of overall uncertainty, we have investigated the robust design problem that aims to minimize the worst-case CRLB over deterministic norm-bounded uncertainties and proved that it can be reformulated as a convex optimization problem. In addition, we have formulated the robust min-max problems corresponding to the uncertainties in individual parameters, namely, the location and the orientation of the VLC receiver. To solve the min-max problem, we have proposed an iterative entropic regularization algorithm, whereby the original problem is transformed into a sequence of convex programs and a grid search is performed over the uncertainty region. Moreover, the problem of total power minimization has been explored under preset accuracy requirements. Simulation results have demonstrated the effectiveness of the optimal power allocation approach in enhancing the localization performance compared to the traditional uniform strategy. Furthermore, the proposed robust power allocation designs have been shown to outperform their non-robust counterparts, especially for large uncertainty regions. Regarding the minimum power consumption problem, power saving gains of $30 \%$ by the optimal strategy have been observed relative to the uniform power allocation approach.

\section{APPENDIX}

A. Definition of $\gamma_{k_{l}, k_{2}}^{(i)}$

$\gamma_{k_{1}, k_{2}}^{(i)}$ in (11) is defined as follows [49]:

$$
\begin{aligned}
\gamma_{k_{1}, k_{2}}^{(i)}= & \left\{\begin{array}{l}
\gamma_{k_{1}, k_{2}}^{(i), \text { syn }}, \text { if synchronous VLP system } \\
\gamma_{k_{1}, k_{2}}^{(i), \text { asy }}, \text { if asynchronous VLP system }
\end{array}\right. \\
\gamma_{k_{1}, k_{2}}^{(i), \text { syn } \triangleq} & \frac{R_{p}^{2}}{\sigma^{2}}\left(E_{2}^{i} \frac{\partial \alpha_{i}}{\partial l_{\mathrm{r}, k_{1}}} \frac{\partial \alpha_{i}}{\partial l_{\mathrm{r}, k_{2}}}+E_{1}^{i} \alpha_{i}^{2} \frac{\partial \tau_{i}}{\partial l_{\mathrm{r}, k_{1}}} \frac{\partial \tau_{i}}{\partial l_{\mathrm{r}, k_{2}}}\right. \\
& \left.-E_{3}^{i} \alpha_{i}\left(\frac{\partial \alpha_{i}}{\partial l_{\mathrm{r}, k_{1}}} \frac{\partial \tau_{i}}{\partial l_{\mathrm{r}, k_{2}}}+\frac{\partial \tau_{i}}{\partial l_{\mathrm{r}, k_{1}}} \frac{\partial \alpha_{i}}{\partial l_{\mathrm{r}, k_{2}}}\right)\right) \\
\gamma_{k_{1}, k_{2}}^{(i), \text { asy } \triangleq} & \frac{R_{p}^{2}}{\sigma^{2}}\left(E_{2}^{i}-\frac{\left(E_{3}^{i}\right)^{2}}{E_{1}^{i}}\right) \frac{\partial \alpha_{i}}{\partial l_{\mathrm{r}, k_{1}}} \frac{\partial \alpha_{i}}{\partial l_{\mathrm{r}, k_{2}}}
\end{aligned}
$$

$$
\begin{aligned}
E_{1}^{i} \triangleq & \int_{0}^{T_{s, i}}\left(\widetilde{s}_{i}^{\prime}(t)\right)^{2} d t \\
E_{2}^{i} \triangleq & \int_{0}^{T_{s, i}}\left(\widetilde{s}_{i}(t)\right)^{2} d t \\
E_{3}^{i} \triangleq & \int_{0}^{T_{s, i}} \widetilde{s}_{i}(t) \widetilde{s}_{i}(t) d t \\
\frac{\partial \tau_{i}}{\partial l_{\mathrm{r}, k}}= & \frac{l_{\mathrm{r}, k}-l_{\mathrm{t}, k}^{i}}{c\left\|\boldsymbol{l}_{\mathrm{r}}-\boldsymbol{l}_{\mathrm{t}}^{i}\right\|} \\
\frac{\partial \alpha_{i}}{\partial l_{\mathrm{r}, k}}= & -\frac{\left(m_{i}+1\right) S}{2 \pi}\left(\frac{\left(\left(\boldsymbol{l}_{\mathrm{r}}-\boldsymbol{l}_{\mathrm{t}}^{i}\right)^{T} \boldsymbol{n}_{\mathrm{t}}^{i}\right)^{m_{i}-1}}{\left\|\boldsymbol{l}_{\mathrm{r}}-\boldsymbol{l}_{\mathrm{t}}^{i}\right\|^{m_{i}+3}}\right. \\
& \times\left(m_{i} n_{\mathrm{t}, k}^{i}\left(\boldsymbol{l}_{\mathrm{r}}-\boldsymbol{l}_{\mathrm{t}}^{i}\right)^{T} \boldsymbol{n}_{\mathrm{r}}+n_{\mathrm{r}, k}\left(\boldsymbol{l}_{\mathrm{r}}-\boldsymbol{l}_{\mathrm{t}}^{i}\right)^{T} \boldsymbol{n}_{\mathrm{t}}^{i}\right) \\
& -\frac{\left(m_{i}+3\right)\left(l_{\mathrm{r}, k}-l_{\mathrm{t}, k}^{i}\right)}{\left\|\boldsymbol{l}_{\mathrm{r}}-\boldsymbol{l}_{\mathrm{t}}^{i}\right\|^{m_{i}+5}} \\
& \left.\times\left(\left(\boldsymbol{l}_{\mathrm{r}}-\boldsymbol{l}_{\mathrm{t}}^{i}\right)^{T} \boldsymbol{n}_{\mathrm{t}}^{i}\right)^{m_{i}}\left(\boldsymbol{l}_{\mathrm{r}}-\boldsymbol{l}_{\mathrm{t}}^{i}\right)^{T} \boldsymbol{n}_{\mathrm{r}}\right)
\end{aligned}
$$

where $\widetilde{s}_{i}^{\prime}(t)$ denotes the derivative of $\widetilde{s}_{i}(t)$.

\section{REFERENCES}

[1] H. Burchardt, N. Serafimovski, D. Tsonev, S. Videv, and H. Haas, "VLC: Beyond point-to-point communication," IEEE Commun. Mag., vol. 52, no. 7, pp. 98-105, Jul. 2014.

[2] P. H. Pathak, X. Feng, P. Hu, and P. Mohapatra, "Visible light communication, networking, and sensing: A survey, potential and challenges," IEEE Commun. Surveys Tuts., vol. 17, no. 4, pp. 2047-2077, 4th Quart., 2015.

[3] A. Jovicic, J. Li, and T. Richardson, "Visible light communication: Opportunities, challenges and the path to market," IEEE Commun. Mag., vol. 51, no. 12, pp. 26-32, Dec. 2013.

[4] D. Karunatilaka, F. Zafar, V. Kalavally, and R. Parthiban, "LED based indoor visible light communications: State of the art," IEEE Commun. Surveys Tuts., vol. 17, no. 3, pp. 1649-1678, Aug. 2015.

[5] J. Armstrong, Y. A. Sekercioglu, and A. Neild, "Visible light positioning: A roadmap for international standardization," IEEE Commun. Mag., vol. 51, no. 12, pp. 68-73, Dec. 2013.

[6] C. Gavrincea, J. Baranda, and P. Henarejos, "Rapid prototyping of standard-compliant visible light communications system," IEEE Commun. Mag., vol. 52, no. 7, pp. 80-87, Jul. 2014.

[7] L. Grobe et al., "High-speed visible light communication systems," IEEE Commun. Mag., vol. 51, no. 11, pp. 60-66, Dec. 2013.

[8] K. Panta and J. Armstrong, "Indoor localisation using white LEDs," Electron. Lett, vol. 48, no. 4, pp. 228-230, 2012.

[9] H.-S. Kim, D.-R. Kim, S.-H. Yang, Y.-H. Son, and S.-K. Han, "An indoor visible light communication positioning system using a RF carrier allocation technique," J. Lightw. Technol., vol. 31, no. 1, pp. 134-144, Jan. 1, 2013.

[10] Z. Ghassemlooy, L. N. Alves, S. Zvanovec, and M.-A. Khalighi, Visible Light Communications: Theory and Applications. Boca Raton, FL, USA: CRC Press, 2017.

[11] T. Komine and M. Nakagawa, "Fundamental analysis for visiblelight communication system using LED lights," IEEE Trans. Consum. Electron., vol. 50, no. 1, pp. 100-107, Feb. 2004.

[12] L. Li, P. Hu, C. Peng, G. Shen, and F. Zhao, "Epsilon: A visible light based positioning system," in Proc. 11th USENIX Symp. Networked Syst. Design Implement. (NSDI), Seattle, WA, USA, Apr. 2014, pp. 331-343.

[13] W. Zhang, M. I. S. Chowdhury, and M. Kavehrad, "Asynchronous indoor positioning system based on visible light communications," Opt. Eng. vol. 53, no. 4, p. 045105, 2014.

[14] S.-H. Yang, E.-M. Jung, and S.-K. Han, "Indoor location estimation based on LED visible light communication using multiple optical receivers," IEEE Commun. Lett., vol. 17, no. 9, pp. 1834-1837, Sep. 2013.

[15] K. Ying, H. Qian, R. J. Baxley, and S. Yao, "Joint optimization of precoder and equalizer in MIMO VLC systems," IEEE J. Sel. Areas Commun., vol. 33, no. 9, pp. 1949-1958, Sep. 2015. 
[16] R. Wang et al., "Linear transceiver designs for MIMO indoor visible light communications under lighting constraints," IEEE Trans. Commun. vol. 65 , no. 6, pp. 2494-2508, Jun. 2017.

[17] B. Li, R. Zhang, W. Xu, C. Zhao, and L. Hanzo, "Joint dimming control and transceiver design for MIMO-aided visible light communication," IEEE Commun. Lett., vol. 20, no. 11, pp. 2193-2196, Nov. 2016.

[18] Q. Gao, C. Gong, and Z. Xu, "Joint transceiver and offset design for visible light communications with input-dependent shot noise," IEEE Trans. Wireless Commun., vol. 16, no. 5, pp. 2736-2747, May 2017.

[19] K.-H. Park, Y.-C. Ko, and M.-S. Alouini, "On the power and offset allocation for rate adaptation of spatial multiplexing in optical wireless MIMO channels," IEEE Trans. Commun., vol. 61, no. 4, pp. 1535-1543, Apr. 2013.

[20] D. Bykhovsky and S. Arnon, "Multiple access resource allocation in visible light communication systems," J. Lightw. Technol., vol. 32, no. 8, pp. 1594-1600, Apr. 15, 2014

[21] C. Gong, S. Li, Q. Gao, and Z. Xu, "Power and rate optimization for visible light communication system with lighting constraints," IEEE Trans. Signal Process., vol. 63, no. 16, pp. 4245-4256, Aug. 2015.

[22] X. Ling, J. Wang, X. Liang, Z. Ding, and C. Zhao, "Offset and power optimization for DCO-OFDM in visible light communication systems," IEEE Trans. Signal Process., vol. 64, no. 2, pp. 349-363, Jan. 2016.

[23] Y. S. Eroğlu, I. Güvenç, A. Şahin, Y. Yapıcı, N. Pala, and M. Yü, "Multielement VLC networks: LED assignment, power control, and optimum combining," IEEE J. Sel. Areas Commun., vol. 36, no. 1, pp. 121-135, Jan. 2017.

[24] R. Jiang, Z. Wang, Q. Wang, and L. Dai, "Multi-user sum-rate optimization for visible light communications with lighting constraints," J. Lightw. Technol., vol. 34, no. 16, pp. 3943-3952, Aug. 15, 2016.

[25] X. Zhang, Q. Gao, C. Gong, and Z. Xu, "User grouping and power allocation for NOMA visible light communication multi-cell networks," IEEE Commun. Lett., vol. 21, no. 4, pp. 777-780, Apr. 2017.

[26] R. Jiang, Q. Wang, H. Haas, and Z. Wang, "Joint user association and power allocation for cell-free visible light communication networks," IEEE J. Sel. Areas Commun., vol. 36, no. 1, pp. 136-148, Jan. 2018.

[27] T. V. Pham, H. Le-Minh, and A. T. Pham, "Multi-user visible light communication broadcast channels with zero-forcing precoding," IEEE Trans. Commun., vol. 65, no. 6, pp. 2509-2521, Jun. 2017.

[28] X. Zhang, S. Dimitrov, S. Sinanovic, and H. Haas, "Optimal power allocation in spatial modulation OFDM for visible light communications," in Proc. IEEE 75th Veh. Technol. Conf. (VTC Spring), May 2012, pp. 1-5.

[29] J. Lian and M. Brandt-Pearce, "Multiuser MIMO indoor visible light communication system using spatial multiplexing," J. Lightw. Technol., vol. 35, no. 23, pp. 5024-5033, Dec. 1, 2017.

[30] W. W.-L. Li, Y. Shen, Y. J. Zhang, and M. Z. Win, "Robust power allocation for energy-efficient location-aware networks," IEEE/ACM Trans. Netw., vol. 21, no. 6, pp. 1918-1930, Dec. 2013

[31] Y. Shen, W. Dai, and M. Z. Win, "Power optimization for network localization," IEEE/ACM Trans. Netw., vol. 22, no. 4, pp. 1337-1350, Aug. 2014.

[32] W. Dai, Y. Shen, and M. Z. Win, "Distributed power allocation for cooperative wireless network localization," IEEE J. Sel. Areas Commun., vol. 33, no. 1, pp. 28-40, Jan. 2015.

[33] T. Zhang, C. Qin, A. F. Molisch, and Q. Zhang, "Joint allocation of spectral and power resources for non-cooperative wireless localization networks," IEEE Trans. Commun., vol. 64, no. 9, pp. 3733-3745, Sep. 2016.

[34] J. Chen, W. Dai, Y. Shen, V. K. N. Lau, and M. Z. Win, "Power management for cooperative localization: A game theoretical approach," IEEE Trans. Signal Process., vol. 64, no. 24, pp. 6517-6532, Dec. 2016

[35] A. Shahmansoori, G. Seco-Granados, and H. Wymeersch, "Power allocation for OFDM wireless network localization under expectation and robustness constraints," IEEE Trans. Wireless Commun., vol. 16, no. 3 , pp. 2027-2038, Mar. 2017

[36] W. Dai, Y. Shen, and M. Z. Win, "A computational geometry framework for efficient network localization," IEEE Trans. Inf. Theory, vol. 64, no. 2, pp. 1317-1339, Feb. 2018

[37] W. Dai, Y. Shen, and M. Z. Win, "Energy-efficient network navigation algorithms," IEEE J. Sel. Areas Commun., vol. 33, no. 7, pp. 1418-1430, Jul. 2015.

[38] T. Wang, G. Leus, and L. Huang, "Ranging energy optimization for robust sensor positioning based on semidefinite programming," IEEE Trans. Signal Process., vol. 57, no. 12, pp. 4777-4787, Dec. 2009.

[39] J. Chen, W. Dai, Y. Shen, V. K. N. Lau, and M. Z. Win, "Resource management games for distributed network localization," IEEE J. Sel. Areas Commun., vol. 35, no. 2, pp. 317-329, Feb. 2017.
[40] H. Elgala, R. Mesleh, and H. Haas, "An LED model for intensitymodulated optical communication systems," IEEE Photon. Technol. Lett., vol. 22, no. 11, pp. 835-837, Jun. 1, 2010.

[41] J. Gancarz, H. Elgala, and T. D. C. Little, "Impact of lighting requirements on VLC systems," IEEE Commun. Mag., vol. 51, no. 12, pp. 34-41, Dec. 2013.

[42] A. Tsiatmas, C. P. M. J. Baggen, F. M. J. Willems, J.-P. M. G. Linnartz, and J. W. M. Bergmans, "An illumination perspective on visible light communications," IEEE Commun. Mag., vol. 52, no. 7, pp. 64-71, Jul. 2014.

[43] Y. S. Eroglu, A. Sahin, I. Guvenc, N. Pala, and M. Yuksel, "Multielement transmitter design and performance evaluation for visible light communication," in Proc. IEEE Globecom Workshops, Dec. 2015, pp. $1-6$.

[44] J. Grubor, S. Randel, K.-D. Langer, and J. Walewski, "Broadband information broadcasting using LED-based interior lighting," J. Lightw. Technol., vol. 26, no. 24, pp. 3883-3892, Dec. 15, 2008.

[45] T. Q. Wang, Y. A. Sekercioglu, A. Neild, and J. Armstrong, "Position accuracy of time-of-arrival based ranging using visible light with application in indoor localization systems," J. Lightw. Technol., vol. 31 , no. 20, pp. 3302-3308, Oct. 15, 2013.

[46] A. Goldsmith, Wireless Communications. Cambridge, U.K.: Cambridge Univ. Press, 2005.

[47] S. De Lausnay, L. De Strycker, J.-P. Goemaere, B. Nauwelaers, and N. Stevens, "A survey on multiple access visible light positioning," in Proc. IEEE Int. Conf. Emerg. Technol. Innov. Bus. Pract. Transformation Soc. (EmergiTech), Aug. 2016, pp. 38-42.

[48] J. M. Kahn and J. R. Barry, "Wireless infrared communications," Proc. IEEE, vol. 85, no. 2, pp. 265-298, Feb. 1997.

[49] M. F. Keskin, S. Gezici, and O. Arikan, "Direct and two-step positioning in visible light systems," IEEE Trans. Commun., vol. 66, no. 1, pp. 239-254, Jan. 2018.

[50] A. Şahin, Y. S. Eroğlu, İ. Güvenç, N. Pala, and M. Yüksel, "Hybrid 3-D localization for visible light communication systems," J. Lightw. Technol., vol. 33, no. 22, pp. 4589-4599, Nov. 15, 2015.

[51] H. V. Poor, An Introduction to Signal Detection and Estimation. New York, NY, USA: Springer-Verlag, 1994.

[52] H. L. V. Trees, Detection, Estimation, and Modulation Theory. New York, NY, USA: Wiley, 2004.

[53] T. D. C. Little and H. Elgala, "Adaptation of OFDM under visible light communications and illumination constraints," in Proc. 48th Asilomar Conf. Signals, Syst. Comput., Nov. 2014, pp. 1739-1744.

[54] H. Ma, L. Lampe, and S. Hranilovic, "Coordinated broadcasting for multiuser indoor visible light communication systems," IEEE Trans. Commun., vol. 63, no. 9, pp. 3313-3324, Sep. 2015.

[55] L. Chen, B. Krongold, and J. Evans, "Theoretical characterization of nonlinear clipping effects in IM/DD optical OFDM systems," IEEE Trans. Commun., vol. 60, no. 8, pp. 2304-2312, Aug. 2012.

[56] E. F. Schubert, Light-Emitting Diodes. Cambridge, U.K.: Cambridge Univ. Press, 2003

[57] A. Pandharipande and D. Caicedo, "Adaptive illumination rendering in LED lighting systems," IEEE Trans. Syst., Man, Cybern., Syst., vol. 43 , no. 5, pp. 1052-1062, Sep. 2013.

[58] S. Boyd and L. Vandenberghe, Convex Optimization. Cambridge, U.K.: Cambridge Univ. Press, 2004.

[59] M. Grant and S. Boyd. (Mar. 2014). CVX: MATLAB Software for Disciplined Convex Programming, Version 2.1. [Online]. Available: http://cvxr.com/cvx

[60] Y. Eldar, A. Ben-Tal, and A. Nemirovski, "Robust mean-squared error estimation in the presence of model uncertainties," IEEE Trans. Signal Process., vol. 53, no. 1, pp. 168-181, Jan. 2005.

[61] M. B. Shenouda and T. N. Davidson, "On the design of linear transceivers for multiuser systems with channel uncertainty," IEEE J. Sel. Areas Commun., vol. 26, no. 6, pp. 1015-1024, Aug. 2008.

[62] J. Wang and D. P. Palomar, "Worst-case robust MIMO transmission with imperfect channel knowledge," IEEE Trans. Signal Process., vol. 57, no. 8, pp. 3086-3100, Aug. 2009.

[63] Y. Hu and G. Leus, "Robust differential received signal strengthbased localization," IEEE Trans. Signal Process., vol. 65, no. 12, pp. 3261-3276, Jun. 2017.

[64] A. Mostafa and L. Lampe, "Optimal and robust beamforming for secure transmission in MISO visible-light communication links," IEEE Trans. Signal Process., vol. 64, no. 24, pp. 6501-6516, Dec. 2016.

[65] A. Ben-Tal and A. Nemirovski, Lectures on Modern Convex Optimization. Philadelphia, PA, USA: SIAM, 2001. [Online]. Available: http://epubs.siam.org/doi/abs/10.1137/1.9780898718829 
[66] S. Boyd, L. El Ghaoui, E. Feron, and V. Balakrishnan, Linear Matrix Inequalities in System and Control Theory. Philadelphia, PA, USA: SIAM, 1994.

[67] L. Vandenberghe and S. Boyd, "Semidefinite programming," SIAM Rev., vol. 38, no. 1, pp. 49-95, 1996.

[68] J. Löfberg, "YALMIP: A toolbox for modeling and optimization in MATLAB," in Proc. CACSD Conf., Taipei, Taiwan, 2004, pp. 284-289.

[69] Z.-Q. Luo, W.-K. Ma, A. M.-C. So, Y. Ye, and S. Zhang, "Semidefinite relaxation of quadratic optimization problems," IEEE Signal Process. Mag., vol. 27, no. 3, pp. 20-34, May 2010.

[70] E. Xu, Z. Ding, and S. Dasgupta, "Source localization in wireless sensor networks from signal time-of-arrival measurements," IEEE Trans. Signal Process., vol. 59, no. 6, pp. 2887-2897, Jun. 2011.

[71] R. L. Sheu and J. Y. Lin, "Solving continuous min-max problems by an iterative entropic regularization method," J. Optim. Theory Appl., vol. 121, no. 3, pp. 597-612, 2004.

[72] H. M. Soliman and A. Leon-Garcia, "Game-theoretic demand-side management with storage devices for the future smart grid," IEEE Trans. Smart Grid, vol. 5, no. 3, pp. 1475-1485, May 2014.

[73] I. Din and H. Kim, "Energy-efficient brightness control and data transmission for visible light communication," IEEE Photon. Technol. Lett., vol. 26, no. 8, pp. 781-784, Apr. 15, 2014.

[74] E. Masazade, R. Niu, P. K. Varshney, and M. Keskinoz, "Energy aware iterative source localization for wireless sensor networks," IEEE Trans. Signal Process., vol. 58, no. 9, pp. 4824-4835, Sep. 2010.

[75] M. F. Keskin and S. Gezici, "Comparative theoretical analysis of distance estimation in visible light positioning systems," J. Lightw. Technol., vol. 34, no. 3, pp. 854-865, Feb. 1, 2016.

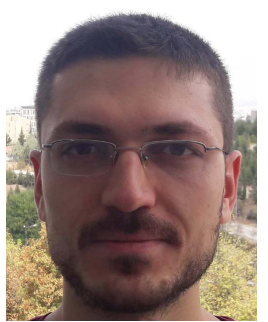

Musa Furkan Keskin received the B.S. and M.S. degrees from the Department of Electrical and Electronics Engineering, Bilkent University, Ankara, Turkey, in 2010 and 2012, respectively. Currently, he is working towards the Ph.D. degree in the same department. His research interests lie in the fields of wireless localization, signal processing, and visible light communications.

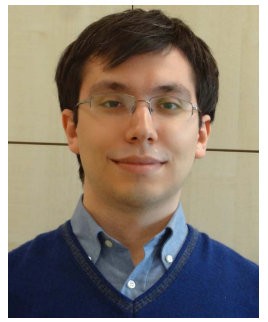

Ahmet Dundar Sezer was born in 1989 in Kütahya, Turkey. He received the B.S. and M.S. degrees in electrical and electronics engineering from Bilkent University, Ankara, Turkey, in 2011 and 2013, respectively, where he is currently pursuing the $\mathrm{Ph} . \mathrm{D}$. degree. His current research interests include signal processing, wireless communications, and optimization.

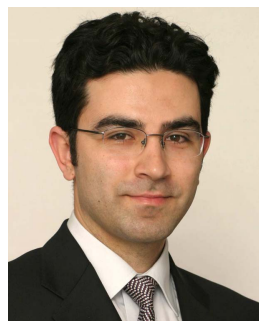

Sinan Gezici (S'03-M'06-SM'11) received the B.S. degree from Bilkent University, Turkey, in 2001, and the Ph.D. degree in electrical engineering from Princeton University in 2006. From 2006 to 2007, he was with Mitsubishi Electric Research Laboratories, Cambridge, MA, USA. Since 2007, he has been with the Department of Electrical and Electronics Engineering, Bilkent University, where he is currently a Professor. His research interests are in the areas of detection and estimation theory, wireless communications, and localization systems. Among his publications in these areas is the book Ultra-wideband Positioning Systems: Theoretical Limits, Ranging Algorithms, and Protocols (Cambridge University Press, 2008). He was an Associate Editor of the IEEE TRANSACTIONS ON COMMUNICATIONS, the IEEE WIRELESS COMMUNICATIONS LETTERS, and the Journal of Communications and Networks. 